\title{
Facilitating safe patient transition of care: A qualitative systematic review
}

\author{
Susan E. Puls ${ }^{1}$, Kerrie S. Guerrero ${ }^{1}$, Dorothy A. Andrew ${ }^{1}$ \\ The University of Texas Health Science Center School of Nursing, Houston, Texas, USA. \\ Correspondence: Susan E. Puls. Address: 1819 Wichita Street, Houston, Texas 77004, USA. Email: \\ sepuls59@gmail.com \\ Received: February 7, 2014 \\ Accepted: March 28, 2014 \\ Online Published: April 17, 2014 \\ DOI : 10.5430/jnep.v4n6p37 \\ URL: http://dx.doi.org/10.5430/jnep.v4n6p37
}

\section{Abstract}

Background: Failure to appropriately plan for a safe and effective transition to the next level of care leads to greater use of hospital and emergency services, often measured by rates of readmission. Despite a focus to develop programs to reduce readmissions, the 30-day all-cause readmission rate for Medicare patients in 2011 remained essentially unchanged.

Purpose: The objective of this qualitative systematic review was to synthesize the evidence for interventions aimed at reducing readmissions through a transition of care program.

Methods: We searched PubMed and Medline (OVID) with search terms including home care services, continuity of patient care, patient discharge, patient-centered care, health planning, and patient readmission. Selection criteria included quantitative studies, qualitative studies, and expert opinion articles in which a transition of care intervention, was implemented. The outcome of interest was readmission rates.

Results: Thirty-three articles met inclusion criteria. The data were synthesized into two categories: primary studies in which the readmission rate was measured as an outcome, and studies that systematically reviewed interventions aimed at improving the discharge process. In all studies reviewed, a transitional care intervention resulted in a statistically significant reduction in readmission rate, or a rate trending lower, or the rate remained the same. Several studies evaluating an intervention occurring during and after hospitalization demonstrated significant results.

Conclusion: There is value in reconfiguring discharge processes toward interventions that are more likely to reduce readmissions. The discharge process should incorporate a multidisciplinary, multicomponent transition of care intervention that involves hospital and home-care follow-up.

\section{Key words}

Transitional care, Patient-centered care, Health planning, Readmissions

\section{I ntroduction}

Mounting evidence is demonstrating that older patients with complex care needs are particularly vulnerable to experiencing serious problems in quality of care when transitioning between different health care settings. Qualitative studies have consistently shown that patients are often unprepared to assume self-management of their care as they transition to the next care setting ${ }^{[1]}$. Common reasons cited are that patients often receive conflicting advice regarding 
illness management, have difficulty accessing health care practitioners who are knowledgeable about their plan of care, and had minimal input into their own plan of care. Quantitative studies revealed that quality and safety are compromised when patients are transitioned between different settings owing to high rates of medication errors, incomplete or inaccurate information transfer, and lack of appropriate follow-up care ${ }^{[1]}$. As a result, poorly executed care transition leads to greater use of hospital and emergency services, which is often measured in terms of increased rates of readmission and which translates into increased health care costs.

\subsection{Background information}

Hospitals and health systems are facing two significant transitions with the move toward population management and value-based purchasing. Organizations will need to create innovative care delivery models to achieve and sustain new quality benchmarks for episodes of care and care management. In addition, they must also prepare for changing payment models that shift risk onto providers ${ }^{[2]}$.

An unplanned readmission to the hospital within 30 days of discharge is seen as a failure by the health care team to appropriately plan for a safe and effective transition to the next level of care. The all-cause readmission rate within 30 days for Medicare patients in 2011 remained high at a national average of $19.3 \%{ }^{[3]}$. The Centers for Medicare and Medicaid Services (CMS) have developed a strategy to improve the quality of care provided to the Medicare population and reduce health care costs by shifting to improving payment for quality. In 2011, acute inpatient facilities received $24 \%$ of all Medicare dollars spent ${ }^{[4]}$. As Medicare has shifted to a pay for performance strategy, a readmission rate higher than the national average for heart failure, pneumonia, or acute myocardial infarction will result in a penalty. Starting in 2013, this penalty is up to $1 \%$ of all inpatient reimbursement received by the hospital ${ }^{[3]}$.

At the time of hospital discharge, many patients are at increased risk from the combination of shorter stays, increased severity of illness, and more complex discharge plans ${ }^{[5]}$. Often, the current discharge planning process in acute inpatient care facilities includes multiple disciplines providing education for patients with a paper copy of instructions. Unit-based case managers and social workers provide discharge planning for patients identified as high risk. These professionals arrange post-discharge placements, durable medical equipment, or home health services. The unit-based registered nurse assumes the responsibility to complete the final check of each discipline and to provide documents on diagnosis, medication reconciliation, and follow-up care. The patient leaves the facility with a mass of papers and instructions that are usually provided on the day of discharge and without confirmation of understanding.

\subsection{Transitional care}

Transitional care is a set of interventions designed to coordinate the care during the movement between health care settings. This process is intentional, is clearly defined with expectations and accountability, and focuses on the needs of the patient and caregiver ${ }^{[6]}$. The interventions may be provided at three different time intervals. An intervention before admission could be an educational class or clinic visit to discuss the hospital and discharge plan. During the hospitalization, the intervention is with a responsible expert or team who assesses needs and develops the plan for care required after discharge. The third time interval is after discharge. This includes telephone follow-up calls and home visits to reinforce teaching as well as to provide support to the patient and caregiver after discharge.

According to a report by the Health Care Advisory Board ${ }^{[2]}$, the top $12 \%$ of Medicare beneficiaries with multiple chronic illnesses account for $43 \%$ of total spending. Focusing efforts on high-risk patients allows organizations to allocate limited resources in such a way as to maximize outcomes and achieve specific care management objectives. In this complex environment, hospitals are asking for evidence of plans that lead to a reduction of readmissions while increasing patient satisfaction and quality of care. Research into the effectiveness of care transition interventions has shown several promising models demonstrating significance in reducing readmission rates, decreasing length of stay, and improving patient satisfaction. The objective of this qualitative systematic review was to synthesize the evidence presented in the literature on transition of care interventions and their effectiveness at reducing readmission rates. 


\section{Methods}

\subsection{Data sources}

PubMed and Medline (OVID) were initially searched for articles and studies published between these databases' inception and March 2013. Medical Subject Headings (MeSH) and text terms were used, which included home care services, continuity of patient care, patient discharge, patient-centered care, health planning, patient readmission, and adult. The reference lists in the studies were also reviewed for potential additional studies missed in the database search. The initial search appeared to lack articles written by Eric A. Coleman, MD, who is well known for his research and work in care transitions. Therefore, a second database search was conducted by adding the search term "Coleman" to retrieve his articles. All search hits were entered into RefWorks (RefWorks-COS, Bethesda, MD) and duplicates were removed, leaving 256 articles with which to begin the study selection process.

\subsection{Study selection}

Selection criteria included studies written in English in which a transition of care intervention, including a nursing component, was implemented before, during, or after hospitalization to adult patients hospitalized in an acute care setting who were being discharged home. The outcome measure of interest was the readmission rate. Articles considered included literature reviews, both quantitative and qualitative primary studies, and reports containing expert opinion. Once the selection criteria were finalized, a two-stage inclusion process was applied. Each of the authors participated equally in the screening and review process for both stages by independently reviewing the studies. Article inclusion and exclusion were discussed as a group to achieve consensus on articles selected for inclusion. To control for risk of bias, another member of the group reviewed any study in which the decision for exclusion or inclusion was in question.

The titles and abstracts of articles were reviewed in the first stage of screening to determine whether they met the inclusion criteria. This initial screening eliminated 135 articles. Reasons for exclusion varied and included wrong population (postpartum, psychiatric, transplant), wrong age (neonate, pediatric), wrong setting (hospice, skilled nursing facility, emergency department), and studies employing an intervention provided by a non-nurse clinician (physical therapist, pharmacist, or primary care provider [PCP]). In addition, any articles in which the title or abstract lacked enough information to confidently determine relevance for inclusion were kept for further review in the second stage.

The second stage of screening was an examination of the full text of the 121 remaining studies and articles. The same inclusion and exclusion criteria used in the initial screening were applied to determine relevance for inclusion. By use of the same exclusion criteria identified in the first stage, 22 articles were excluded for wrong population, setting, or intervention. In addition, 23 studies were excluded owing to the full text being unavailable or not being written in English. A total of 27 articles had a study design that was weak or poor, unclear, or did not measure the correct outcome measure; these 27 articles were also excluded. After completion of the two-stage screening process and group consensus, 33 articles met the inclusion criteria for final review and synthesis of evidence. A flow diagram of the literature selection is depicted in Figure 1.

\subsection{General characteristics}

The characteristics of the final selection of articles can be seen in Table 1 (meta-reviews and systematic reviews) and Table 2 (primary studies). These studies were published in 1993 or later, had sample sizes ranging from 30 to 3,998, and varied by study type including systematic reviews, randomized controlled trials (RCTs), quasi-experimental studies, cohort studies, and those categorized as observational, descriptive, or qualitative. The main population addressed in the majority of studies consisted of elderly patients with various diagnoses. Some studies were restricted to a specific patient group, such as patients with myocardial infarction, heart failure, or acute stroke. Patients were discharged from acute, general medical, cardiac, and surgical units from various types of hospitals, including urban, tertiary, teaching, and university-affiliated. Besides the outcome of interest for this review, many of the studies reported additional outcomes related to hospital or health care services utilized, continuity of care, patient status, and cost of health services. 


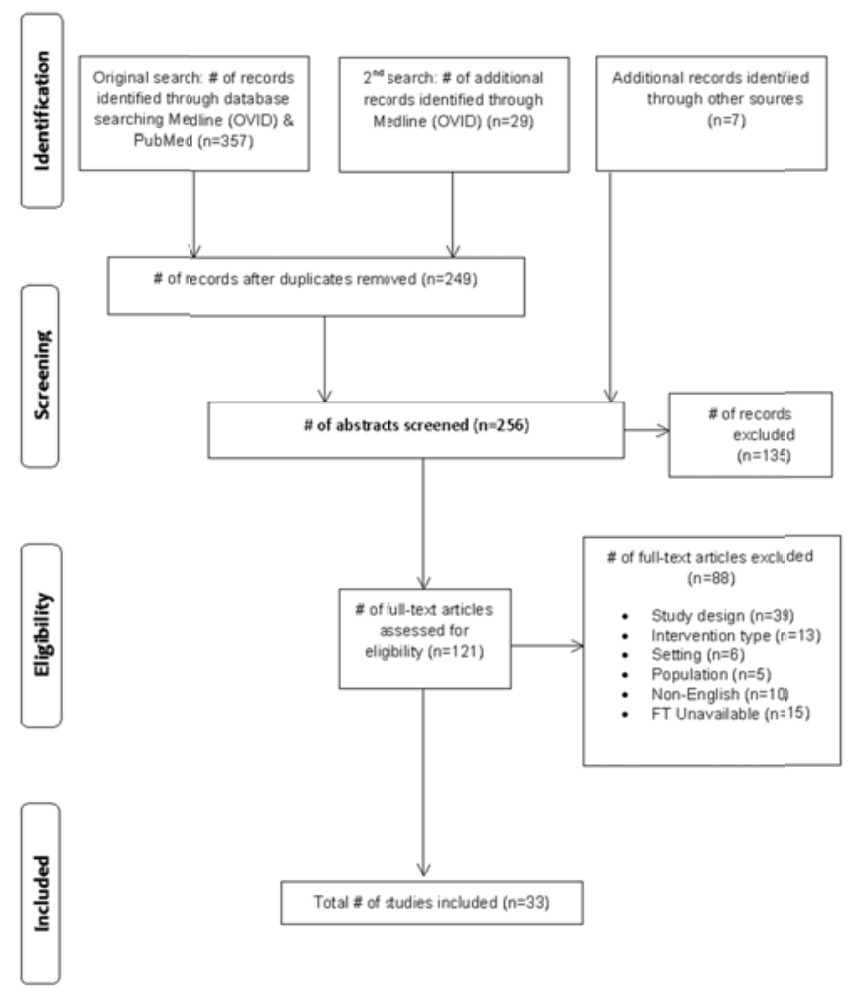

Figure 1. Flow chart of the citations reviewed to achieve the final 33 studies included for synthesis of evidence in this qualitative systematic review

Table 1. Evidence table of systematic and meta-reviews

\begin{tabular}{|c|c|c|c|c|c|}
\hline Review & $\begin{array}{l}\text { Type of } \\
\text { Review }\end{array}$ & $\begin{array}{l}\text { Search } \\
\text { Period }\end{array}$ & $\begin{array}{l}\text { No. \& types } \\
\text { of studies } \\
\text { included }\end{array}$ & Aim & Relevant Findings \\
\hline $\begin{array}{l}\text { Hesselink } \\
\text { et al. } \\
(2012)^{[36]}\end{array}$ & $\begin{array}{l}\text { Systematic } \\
\text { Review of } \\
\text { RCTs } \\
\text { Level I }\end{array}$ & $1990-2011$ & 36 RCTs & $\begin{array}{l}\text { To systematically review } \\
\text { interventions that aim to } \\
\text { improve patient discharge } \\
\text { from hospital to primary } \\
\text { care. }\end{array}$ & $\begin{array}{l}\text { Intervention had to address at least } 1 \text { component aimed to improve } \\
\text { handover of care between hospital \& PCP during hospital DC. } 25 \\
\text { studies had statistically significant effects in favor of the intervention } \\
\text { group. No conclusion re: which interventions have most positive } \\
\text { effects. }\end{array}$ \\
\hline $\begin{array}{l}\text { Jacob \& } \\
\text { Poletick } \\
(2008)^{[38]}\end{array}$ & $\begin{array}{l}\text { Qualitative } \\
\text { Systematic } \\
\text { Review } \\
\text { Level V }\end{array}$ & $1997-2007$ & $\begin{array}{l}10 \text { articles } \\
\text { (RCTs, } \\
\text { quasi-experi } \\
\text { mental, } \\
\text { retrospective } \\
\text { reviews, \& } \\
\text { interpretive } \\
\text { studies) }\end{array}$ & $\begin{array}{l}\text { To assess the effects of } \\
\text { "enhanced discharge } \\
\text { support" for patients } \\
\text { identified as susceptible to } \\
\text { difficult transition when } \\
\text { transitioning from acute } \\
\text { hospital back to the } \\
\text { community. }\end{array}$ & $\begin{array}{l}\text { "Enhanced discharge support" demonstrated a positive effect in } \\
\text { preventing or delaying readmissions for certain diagnoses, such as HF } \\
\text { \& stroke. In addition, those with adequate social support and } \\
\text { confidence in self-care tend to experience fewer readmissions than } \\
\text { those living alone or those who perceive themselves as not ready for } \\
\text { discharge. }\end{array}$ \\
\hline $\begin{array}{l}\text { Linertiva } \\
\text { et al. } \\
(2010)^{[37]}\end{array}$ & $\begin{array}{l}\text { Systematic } \\
\text { Review of } \\
\text { clinical } \\
\text { trials } \\
\text { (randomiz } \\
\text { ed \& } \\
\text { controlled) } \\
\text { Level I }\end{array}$ & $\begin{array}{l}\text { Inception- } \\
2009\end{array}$ & $\begin{array}{l}32 \text { clinical } \\
\text { trials ( } 17 \\
\text { in-hospital } \\
\text { interventions } \\
\& 15 \\
\text { interventions } \\
\text { with home } \\
\text { f/u) }\end{array}$ & $\begin{array}{l}\text { To identify interventions that } \\
\text { effectively reduce the risk of } \\
\text { hospital readmissions in } \\
\text { patients } 75 \text { years and older. }\end{array}$ & $\begin{array}{l}\text { Most of the interventions evaluated did not have any effect on the } \\
\text { readmission of elderly patients; however, those interventions that } \\
\text { included geriatric management and home care components seem to be } \\
\text { more likely to reduce readmissions. }\end{array}$ \\
\hline $\begin{array}{l}\text { Mistiaen } \\
\text { et al. } \\
(2007)^{[34]}\end{array}$ & $\begin{array}{l}\text { Systematic } \\
\text { Meta-revie } \\
\text { w } \\
\text { Level I }\end{array}$ & 1994-2004 & $\begin{array}{l}15 \text { systematic } \\
\text { reviews }\end{array}$ & $\begin{array}{l}\text { Conduct a systematic review } \\
\text { of reviews examining } \\
\text { effectiveness of discharge } \\
\text { interventions aimed to } \\
\text { reduce post-discharge } \\
\text { problems in adults } \\
\text { discharged home from an } \\
\text { acute general care hospital. }\end{array}$ & $\begin{array}{l}\text { Based on these reviews, some evidence exists that some interventions } \\
\text { may have a positive impact, particularly those with educational } \\
\text { components and those that combine pre-discharge and post-discharge } \\
\text { interventions. However, although a statistical significant effect was } \\
\text { occasionally found, most reviews reached no firm conclusions that the } \\
\text { discharge interventions were effective. }\end{array}$ \\
\hline $\begin{array}{l}\text { Scott } \\
(2010)^{[35]}\end{array}$ & $\begin{array}{l}\text { Systematic } \\
\text { Meta-revie } \\
\text { w of } \\
\text { controlled } \\
\text { trials } \\
\text { Level I }\end{array}$ & 1990-2009 & $\begin{array}{l}7 \text { systematic } \\
\text { reviews } \\
\text { published } \\
\text { after } 2000\end{array}$ & $\begin{array}{l}\text { To determine the relative } \\
\text { efficacy of peri-discharge } \\
\text { interventions categorized } \\
\text { into two groups: single } \\
\text { component interventions } \\
\text { implemented before or after } \\
\text { discharge and integrated } \\
\text { multicomponent } \\
\text { interventions, which have } \\
\text { pre- and post-discharge } \\
\text { elements. }\end{array}$ & $\begin{array}{l}\text { Intense self-management and transition coaching of patients at high } \\
\text { risk for readmission and use of home visits or telephone support for HF } \\
\text { patients appear to be the only single-component strategies that } \\
\text { demonstrated evidence of reducing readmission. The multicomponent } \\
\text { studies appeared to show a positive outcome in reducing readmission } \\
\text { rates. }\end{array}$ \\
\hline
\end{tabular}


Table 2. Evidence table of primary studies

\begin{tabular}{|c|c|c|c|c|c|c|c|}
\hline Citation & $\begin{array}{l}\text { Research } \\
\text { Questions/ } \\
\text { Hypothesis }\end{array}$ & $\begin{array}{l}\text { Design/ } \\
\text { Level of } \\
\text { Evidence }\end{array}$ & $\begin{array}{l}\text { Independent } \\
\text { Variables and } \\
\text { Measures }\end{array}$ & $\begin{array}{l}\text { Dependent } \\
\text { Variables } \\
\text { and } \\
\text { Measures } \\
\end{array}$ & $\begin{array}{l}\text { Sample Size } \\
\text { Pop'n }\end{array}$ & Results & Additional Notes \\
\hline $\begin{array}{l}\text { Aguado et al. } \\
(2010)^{[10]}\end{array}$ & $\begin{array}{l}\text { Study the } \\
\text { effectiveness of a } \\
\text { single } \\
\text { home-based } \\
\text { educational } \\
\text { intervention in } \\
\text { patients with } \\
\text { systolic HF }\end{array}$ & $\begin{array}{l}\text { RCT } \\
\text { Level II }\end{array}$ & $\begin{array}{l}\text { Nurse home } \\
\text { visit within } 1 \\
\text { week of } \\
\text { discharge. }\end{array}$ & $\begin{array}{l}\text { Hospital } \\
\text { admissions }\end{array}$ & $\begin{array}{l}\mathrm{N}=106 \\
\text { (admitted to a } \\
\text { teaching } \\
\text { hospital for } \\
\text { HF) }\end{array}$ & $\begin{array}{l}24 \text { month f/u: fewer } \\
\text { unplanned admissions } \\
(p=.000)\end{array}$ & $\begin{array}{l}\text { Additional study measures: ED visits. } \\
69 / 106 \text { patients lost to f/u at } 24 \text { months } \\
\text { leaving } 37 \text { patients in the study. Very } \\
\text { small sample of middle to upper income } \\
\text { patients. } \\
\text { (Intervention: post-discharge) }\end{array}$ \\
\hline $\begin{array}{l}\text { Booth et al. } \\
(2004)^{[7]}\end{array}$ & $\begin{array}{l}\text { Does early } \\
\text { discharge reduce } \\
\text { hospital costs, } \\
\text { increase the } \\
\text { throughput of } \\
\text { patients, and } \\
\text { decrease waiting } \\
\text { list times? }\end{array}$ & $\begin{array}{l}\text { RCT } \\
\text { Level II }\end{array}$ & $\begin{array}{l}\text { Pre-admit } \\
\text { clinic visit, } \\
\text { admission day } \\
\text { of procedure } \\
\text { with early DC, } \\
\text { specialist } \\
\text { nurses } \\
\text { provided } \\
\text { home care }\end{array}$ & $\begin{array}{l}\text { Readmissio } \\
\text { n rates at } 12 \\
\text { weeks }\end{array}$ & $\begin{array}{l}\mathrm{N}=97 \\
\text { patients }\end{array}$ & $\begin{array}{l}\text { Readmission rates at } 12 \\
\text { weeks were similar in the } \\
\text { two groups. }\end{array}$ & $\begin{array}{l}\text { Additional study measures: LOS, } \\
\text { clinical events, costs, \& QOL. } \\
\text { (Intervention: pre-admission and } \\
\text { post-discharge) }\end{array}$ \\
\hline $\begin{array}{l}\text { Bull } \\
(1994)^{[11]}\end{array}$ & $\begin{array}{l}\text { Identify } \\
\text { predictors of } \\
\text { post-DC resource } \\
\text { use, } \\
\text { services used, \& } \\
\text { readmission rates } \\
\text { of elder PTs } \\
\text { receiving } \\
\text { community } \\
\text { services. }\end{array}$ & $\begin{array}{l}\text { Qualitative } \\
\text { Level IV }\end{array}$ & $\begin{array}{l}\text { Elder patients } \\
\text { with caregiver } \\
\text { who received } \\
\text { community } \\
\text { services. }\end{array}$ & $\begin{array}{l}\text { Readmissio } \\
\text { n rates }\end{array}$ & $\begin{array}{l}\mathrm{N}=185 \\
\text { (elder/caregiv } \\
\text { er dyads) }\end{array}$ & $\begin{array}{l}\text { Elders who receive } \\
\text { visiting nurse services are } \\
\text { less likely to be } \\
\text { readmitted to the hospital. }\end{array}$ & $\begin{array}{l}\text { Additional study measures: } \\
\text { Pre-discharge functional ability using } \\
\text { Philadelphia Geriatric Multi-level } \\
\text { Assessment Instrument (PGC-MAI) to } \\
\text { measure elder and caregiver health. The } \\
\text { majority of caregivers were women. } \\
\text { (Invention: post-discharge) }\end{array}$ \\
\hline $\begin{array}{l}\text { Cardozo \& } \\
\text { Steinberg } \\
\left(^{(2010)^{[12]}}\right.\end{array}$ & $\begin{array}{l}\text { The purpose of } \\
\text { this study is to } \\
\text { evaluate a case- } \\
\text { managed } \\
\text { telemedicine } \\
\text { (CMTM) } \\
\text { program and } \\
\text { patient } \\
\text { acceptance, } \\
\text { satisfaction, and } \\
\text { cost. }\end{array}$ & $\begin{array}{l}\text { Observatio } \\
\text { nal } \\
\text { Level VI }\end{array}$ & $\begin{array}{l}\text { Nurse visit up } \\
\text { to } 3 x \text { per } \\
\text { week, home } \\
\text { tele-monitorin } \\
\text { g daily. }\end{array}$ & $\begin{array}{l}60 \text {-day } \\
\text { readmission } \\
\text { rate }\end{array}$ & $\begin{array}{l}\mathrm{N}=851 \\
\text { (patients with } \\
\text { HF, COPD, } \\
\text { DM, or HTN) }\end{array}$ & $\begin{array}{l}\text { 60-day readmission rate } \\
\text { was } 13.9 \% \text { vs. } 56.4 \% \text { that } \\
\text { was reported in a large, } \\
\text { national study of } \\
\text { tele-monitoring. }\end{array}$ & $\begin{array}{l}\text { Additional study measures: Compliance } \\
\text { rates, improvement in 9-quality of care } \\
\text { measures (QCM), satisfaction, \& } \\
\text { mortality. Older primarily minority } \\
\text { patients. The tele-monitoring was well } \\
\text { received by the patients who said it } \\
\text { provided a sense of security; they felt } \\
\text { more involved and would recommend to } \\
\text { their peers. } \\
\text { (Intervention: post-discharge) }\end{array}$ \\
\hline $\begin{array}{l}\text { Chang et al. } \\
(2003){ }_{[9]}\end{array}$ & $\begin{array}{l}\text { The purpose of } \\
\text { the study was to } \\
\text { identify and } \\
\text { analyze risk } \\
\text { factors leading to } \\
\text { readmission } \\
\text { among patients } \\
\text { visited by a DC } \\
\text { Coordinator. }\end{array}$ & $\begin{array}{l}\text { Descriptive } \\
\text { Retrospecti } \\
\text { ve } \\
\text { Level VI }\end{array}$ & $\begin{array}{l}\text { Discharge } \\
\text { Coordinator } \\
\text { interviews } \\
\text { patients while } \\
\text { in the hospital } \\
\text { and develops a } \\
\text { plan. }\end{array}$ & $\begin{array}{l}\text { 14-day } \\
\text { readmission } \\
\text { rate }\end{array}$ & $\begin{array}{l}\mathrm{N}=1079 \\
\text { (patients } \\
\text { discharged } \\
\text { home and } \\
\text { visited by a } \\
\text { Discharge } \\
\text { Coordinator in } \\
\text { the hospital) }\end{array}$ & $\begin{array}{l}67 \text { patients }(5.7 \%) \text { were } \\
\text { readmitted within } 14 \text { days } \\
\text { of discharge ( } 28 \text { patients } \\
\text { were readmitted because } \\
\text { of complications). } \\
\text { Readmission within } 14 \\
\text { days of discharge had } \\
\text { statistically significant } \\
\text { relationships with visits } \\
\text { from social workers; } \\
\text { home-care nurses and } \\
\text { hospice home-care nurses } \\
(P<.05) \text {. }\end{array}$ & $\begin{array}{l}\text { The Discharge Coordinator identified } \\
\text { eight groups of } \\
\text { patients who might need continuing care } \\
\text { after discharge according to the } \\
\text { following criteria: } 2 \text { or more chronic } \\
\text { diseases, cognitive impairment, } \\
\text { readmission, > } 70 \text {, lives alone or in an } \\
\text { institution, bedridden, LOS > } 30 \text { days, } \\
\text { DM or stroke. } \\
\text { (Intervention: during inpatient stay) }\end{array}$ \\
\hline $\begin{array}{l}\text { Coleman et al. } \\
(2004)^{[33]}\end{array}$ & $\begin{array}{l}\text { To test whether } \\
\text { an intervention } \\
\text { designed to } \\
\text { encourage older } \\
\text { patients and their } \\
\text { caregivers to } \\
\text { assert a more } \\
\text { active role during } \\
\text { care transitions } \\
\text { can reduce } \\
\text { rehospitalization } \\
\text { rates. }\end{array}$ & $\begin{array}{l}\text { Quasi-ex- } \\
\text { perimental } \\
\text { Level III }\end{array}$ & $\begin{array}{l}\text { Patients given } \\
\text { tools to } \\
\text { promote a } \\
\text { more active } \\
\text { role in their } \\
\text { care. } \\
\text { Transitional } \\
\text { coach, } \\
\text { post-discharge } \\
\text { home visit. }\end{array}$ & $\begin{array}{l}\text { Post-discha } \\
\text { rge hospital } \\
\text { use at } 30 \\
60 \text {, and } 90 \\
\text { days. }\end{array}$ & $\begin{array}{l}\text { Intervention } \\
\text { group } \mathrm{N}=158 . \\
\text { Comparison } \\
\text { group } \mathrm{N}= \\
1235, \\
\text { administrative } \\
\text { data. }\end{array}$ & $\begin{array}{l}\text { The odds ratio of } \\
\text { readmission in the } \\
\text { intervention group }=0.52 \\
\text { [95\% confidence interval } \\
(\mathrm{CI})=0.28-0.96 \text { at } 30 \\
\text { days], } 0.43(95 \%(\mathrm{CI})= \\
0.25-0.72) \text { at } 90 \text { days. }\end{array}$ & $\begin{array}{l}\text { Additional study measures: Patients' } \\
\text { care experiences. By supporting patients } \\
\text { and caregivers through the transition of } \\
\text { care, the patients were half as likely to } \\
\text { be readmitted. } \\
\text { (Intervention: during inpatient stay and } \\
\text { post-discharge) }\end{array}$ \\
\hline $\begin{array}{l}\text { Coleman et al. } \\
(2005)^{[15]}\end{array}$ & $\begin{array}{l}\text { The objective } \\
\text { was to study the } \\
\text { incidence of and } \\
\text { factors related to } \\
\text { medication } \\
\text { discrepancies. }\end{array}$ & $\begin{array}{l}\text { Descriptive } \\
\text { Level IV }\end{array}$ & $\begin{array}{l}\text { GNP visit } \\
\text { within 24-48 } \\
\text { hr. after } \\
\text { discharge to } \\
\text { assess the pre- } \\
\text { and } \\
\text { post-hospital } \\
\text { medication } \\
\text { regimen. }\end{array}$ & $\begin{array}{l}\text { 30-day } \\
\text { readmission } \\
\text { rate }\end{array}$ & $\begin{array}{l}\mathrm{N}=375 \text { (age } 65 \\
\text { or older } \\
\text { discharged } \\
\text { from the } \\
\text { hospital) }\end{array}$ & $\begin{array}{l}\text { 30-day readmission rate } \\
\text { was higher for patients } \\
\text { experiencing medication } \\
\text { discrepancies }(\mathrm{p}=0.04) \text {. }\end{array}$ & $\begin{array}{l}\text { Additional study measures: Medication } \\
\text { discrepancies. } 14.1 \% \text { of patients had } 1 \\
\text { or more medication discrepancies. } \\
\text { Medication discrepancies were } \\
\text { associated with the total number of } \\
\text { medications taken and the presence of } \\
\text { congestive heart failure. } \\
\text { (Intervention: post-discharge) }\end{array}$ \\
\hline $\begin{array}{l}\text { Cotton et al. } \\
(2000)^{[17]}\end{array}$ & $\begin{array}{l}\text { Compare early } \\
\text { discharge with } \\
\text { home care f/u by } \\
\text { respiratory } \\
\text { nurses and } \\
\text { conventional } \\
\text { hospital } \\
\text { management in } \\
\text { patients with } \\
\text { COPD. }\end{array}$ & $\begin{array}{l}\text { RCT } \\
\text { Level II }\end{array}$ & $\begin{array}{l}\text { Early } \\
\text { discharge and } \\
\text { visit by a } \\
\text { respiratory } \\
\text { nurse on the } \\
\text { day after } \\
\text { discharge. }\end{array}$ & $\begin{array}{l}\text { 30-day } \\
\text { readmission } \\
\text { rate }\end{array}$ & $\mathrm{N}=81$ patients. & $\begin{array}{l}\text { Readmission rates were } \\
\text { similar in the two groups. }\end{array}$ & $\begin{array}{l}\text { Additional study measures: In-hospital } \\
\text { clinical events, number of days spent in } \\
\text { the hospital during } 60 \text { days following } \\
\text { initial admission, and mortality. } \\
\text { The duration of inpatient stay after } \\
\text { readmission was similar in the two } \\
\text { groups. The time to readmission shows } \\
\text { that the early discharge group did not } \\
\text { have a different pattern of readmissions } \\
\text { from the control group. } \\
\text { (Intervention: post-discharge) }\end{array}$ \\
\hline $\begin{array}{l}\text { Feldman et al. } \\
(2011)_{[20]}\end{array}$ & $\begin{array}{l}\text { Describe \& } \\
\text { compare 1-year } \\
\text { outcomes in men } \\
\text { and women } \\
\text { attending HF } \\
\text { clinics. }\end{array}$ & $\begin{array}{l}\text { Cohort } \\
\text { Observatio } \\
\text { nal } \\
\text { Level IV }\end{array}$ & $\begin{array}{l}\text { Follow-up at a } \\
\text { HF clinic after } \\
\text { hospital } \\
\text { discharge for } \\
\text { newly } \\
\text { diagnosed } \\
\text { patients. }\end{array}$ & $\begin{array}{l}\text { Hospital } \\
\text { admissions } \\
\text { after } 12 \text { mo. }\end{array}$ & $\begin{array}{l}\mathrm{N}=531 \\
\text { (patients from } \\
6 \text { HF clinics) }\end{array}$ & $\begin{array}{l}\text { After } 12 \text { mo of } \mathrm{f} / \mathrm{u} \text { in the } \\
\text { HF clinic, } 14.32 \% \text { were } \\
\text { hospitalized (vs. } 29.4 \% \\
\text { within } 6 \text { mo preceding } \\
\text { admission to the clinic). } \\
\text { No differences noted } \\
\text { between men and women } \\
\text { for readmissions. }\end{array}$ & $\begin{array}{l}\text { Additional study measures: mortality, } \\
\text { disease progression, and ED visits. } \\
\text { (Intervention: post-discharge) }\end{array}$ \\
\hline $\begin{array}{l}\text { Kwok et al. } \\
(2008)^{[27]}\end{array}$ & $\begin{array}{l}\text { Will a } \\
\text { community } \\
\text { nurse-supported } \\
\text { hosp. discharge } \\
\text { program (CNP) } \\
\text { prevent hospital } \\
\text { readmissions } \\
\text { among older } \\
\text { patients with HF? }\end{array}$ & $\begin{array}{l}\text { RCT } \\
\text { Level II }\end{array}$ & $\begin{array}{l}\text { Interaction } \\
\text { with a } \\
\text { community } \\
\text { nurse - visit } \\
\text { before DC, } \\
\text { within } 7 \text { days } \\
\text { of DC, weekly } \\
\text { for } 4 \text { wk, then } \\
\text { monthly. }\end{array}$ & $\mathrm{RR}$ at $6 \mathrm{mo}$. & $\begin{array}{l}\mathrm{N}=105 \text { (age } \\
60 \text { yr or older } \\
\text { with HF \& } \\
\text { history of } \\
\text { hospital } \\
\text { admission(s) } \\
\text { in previous } \\
\text { year) }\end{array}$ & $\begin{array}{l}\text { At } 6 \text { mo, the RRs were } \\
\text { not significantly different } \\
\text { ( } 46 \% \text { vs. } 57 \% \text { in CG; } \\
p=0.233) \text {, but median } \\
\text { number of RR tended to } \\
\text { be lower in the IG ( } 0 \text { vs. } 1 \\
\text { in CG; } p=0.057)\end{array}$ & $\begin{array}{l}\text { Additional study measures: 6-min } \\
\text { walking distance, London Handicap } \\
\text { Scale, \& \$\$. Effective in preserving } \\
\text { independence \& probably effective in } \\
\text { reducing the number of unplanned } \\
\text { re-admissions. } \\
\text { (Intervention: during inpatient stay and } \\
\text { post-discharge) }\end{array}$ \\
\hline
\end{tabular}


Table 2. (Continued.)

\begin{tabular}{|c|c|c|c|c|c|c|c|}
\hline Citation & $\begin{array}{l}\text { Research } \\
\text { Questions/ } \\
\text { Hypothesis }\end{array}$ & $\begin{array}{l}\text { Design/ } \\
\text { Level of } \\
\text { Evi- } \\
\text { dence }\end{array}$ & $\begin{array}{l}\text { Independent } \\
\text { Variables and } \\
\text { Measures }\end{array}$ & $\begin{array}{l}\text { Dependent } \\
\text { Variables } \\
\text { and } \\
\text { Measures } \\
\end{array}$ & $\begin{array}{l}\text { Sample Size } \\
\text { Pop'n }\end{array}$ & Results & Additional Notes \\
\hline $\begin{array}{l}\operatorname{Lim} e t \\
\text { al. } \\
(2003)^{[8]}\end{array}$ & $\begin{array}{l}\text { Does a PAC } \\
\text { (Post-Acute } \\
\text { Care) } \\
\text { intervention } \\
\text { benefit older } \\
\text { patients after } \\
\text { discharge from } \\
\text { the hospital? }\end{array}$ & $\begin{array}{l}\text { RCT } \\
\text { Level II }\end{array}$ & $\begin{array}{l}\text { PAC } \\
\text { intervention: } \\
\text { PAC } \\
\text { Coordinator } \\
\text { develops a DC } \\
\text { plan including } \\
\text { CM. }\end{array}$ & $\begin{array}{l}\text { RR within } 6 \\
\text { months. }\end{array}$ & $\begin{array}{l}\mathrm{N}=654(65 \\
\text { years and } \\
\text { older requiring } \\
\text { community } \\
\text { services after } \\
\text { discharge). }\end{array}$ & $\begin{array}{l}\text { No difference in } \\
\text { unplanned RR. IG group } \\
\text { used statistically } \\
\text { significantly fewer } \\
\text { hospital days in } 6 \text { months } \\
\text { after discharge }(p=.01) .\end{array}$ & $\begin{array}{l}\text { Additional study measures: QOL \& Caregiver } \\
\text { stress, mortality, hospital \& community service } \\
\text { utilization, and health-related service \$\$. PAC } \\
\text { Coordinators coordinated discharge planning } \\
\text { and had a good working knowledge of local } \\
\text { community services \& knew how to obtain such } \\
\text { services on short notice. } \\
\text { (Intervention: during inpatient stay) }\end{array}$ \\
\hline $\begin{array}{l}\text { Martin et } \\
\text { al. } \\
(1994)^{[24]}\end{array}$ & $\begin{array}{l}\text { Will patients } \\
\text { receiving HTT } \\
\text { (Home } \\
\text { Treatment Team) } \\
\text { after hospital DC } \\
\text { have fewer } \\
\text { readmissions at } 6 \\
\text { \& } 12 \text {-wk? }\end{array}$ & $\begin{array}{l}\text { RCT } \\
\text { Level II }\end{array}$ & $\begin{array}{l}\text { HTT (hospital } \\
\text { discharge } \\
\text { team for } \\
\text { elderly PTs } \\
\text { providing } \\
\text { practical help } \\
\text { at home \& } \\
\text { promoting } \\
\text { independence } \\
\text { for up to } 6 \text { wk } \\
\text { post DC) }\end{array}$ & $\begin{array}{l}\text { RR at } 6 \& \\
12 \text { weeks. }\end{array}$ & $\begin{array}{l}\mathrm{N}=54 \\
\text { (elderly } \\
\text { patients } \\
\text { judged to be at } \\
\text { risk) }\end{array}$ & $\begin{array}{l}\text { Fewer HTT PTs were } \\
\text { readmitted @ } 6 \text { and } 12 \\
\text { wk }(p<.05) \text {. More HTT } \\
\text { PTs were home @ } 6 \text { wk } \\
(p<.05), 12 \text { wk }(p<.05) \text {, } \\
\& 12 \text { months }(p<.05) . \\
\text { HTT group spent fewer } \\
\text { days in hospital than CG } \\
\text { during } 12 \text { wk. }\end{array}$ & $\begin{array}{l}\text { Additional study measures: \# of PTs at home at } 6 \\
\text { wk, } 12 \text { wk, } \& 12 \text { mo; \# of hospital days vs. home } \\
\text { days. The HTT worker visited the patient up to } \\
\text { 3x day between } 8 \text { am } \& \text { pm for up to } 6 \text { weeks. } \\
\text { Tasks performed included personal care and } \\
\text { home assistance excluding tasks generally } \\
\text { requiring an RN. Some of the readmissions } \\
\text { occurred very early after DC \& in most there was } \\
\text { an acute event, such as a fall. } \\
\text { (Intervention: during inpatient stay and } \\
\text { post-discharge) }\end{array}$ \\
\hline $\begin{array}{l}\text { Melton } \\
\text { et al. } \\
(2012)^{[18]}\end{array}$ & $\begin{array}{l}\text { To determine if } \\
\text { post-discharge } \\
\text { telephonic CM } \\
\text { reduces hospital } \\
\text { readmissions for } \\
\text { high-risk PTs. }\end{array}$ & $\begin{array}{l}\text { RCT } \\
\text { Level II }\end{array}$ & $\begin{array}{l}\text { Telephonic } \\
\text { CM within } 24 \\
\text { hr of DC } \\
\text { prioritized } \\
\text { based on } \\
\text { health risk } \\
\text { order. }\end{array}$ & $\begin{array}{l}\text { RR @ } 30 \& \\
60 \text { days; RR } \\
\text { per } 1000\end{array}$ & $\begin{array}{l}\mathrm{N}=3998 \\
\text { (adults with } 1 \\
\text { of } 3 \text { major } \\
\text { diagnoses, } 3 \text { or } \\
\text { more days } \\
\text { LOS, private } \\
\text { insurance) }\end{array}$ & $\begin{array}{l}30 \text {-day RR for IG was } \\
5.7 \% \text { vs. } 7.3 \% \text { for CG ( } p \\
<.05) ; 60-\text { day RR for IG } \\
\text { was } 7.5 \% \text { vs. } 9.6 \% \text { for } \\
\text { CG }(p<.05) \text {; RR per } \\
1000 \text { for IG was } 230 \text { vs. } \\
261 \text { for CG }(p<.005)\end{array}$ & $\begin{array}{l}\text { IG received } 2 \text { attempted phone calls within } 24 \mathrm{hr} \\
\text { after DC. All calls were made in descending } \\
\text { health risk order based on risk assessment score } \\
\text { so that outreach was administered to PTs with } \\
\text { greatest likelihood of readmission. } \\
\text { (Intervention: post-discharge) }\end{array}$ \\
\hline $\begin{array}{l}\text { Miranda } \\
\text { et al. } \\
\text { (2002) }\end{array}$ & $\begin{array}{l}\text { Will } \\
\text { implementation } \\
\text { of a cross-site EB } \\
\text { approach to HF } \\
\text { improve } \\
\text { outcomes? }\end{array}$ & $\begin{array}{l}\text { Descripti } \\
\text { ve } \\
\text { Level VI }\end{array}$ & $\begin{array}{l}\text { Implementa-ti } \\
\text { on of } \\
\text { disease-specifi } \\
\text { c guidelines } \\
\text { for HF } \\
\text { management, } \\
\text { including pt. } \\
\text { education, \& } \\
\text { post DC phone } \\
\text { f/u. }\end{array}$ & $\begin{array}{l}\text { RR for } \\
\text { HF-related } \\
\text { conditions } \\
\text { within } 30 \text { \& } \\
90 \text { days. }\end{array}$ & $\begin{array}{l}\text { HF patients } \\
\text { cared for } \\
\text { within the } \\
\text { Covenant } \\
\text { Healthcare } \\
\text { System in } \\
\text { Southeast } \\
\text { Wisconsin. }\end{array}$ & $\begin{array}{l}30 \text {-day RR decreased } \\
\text { from } 14 \% \text { to } 6.8 \% \text {, and } \\
90 \text {-day RR decreased } \\
\text { from } 17.5 \% \text { to } 12.5 \% \\
\text { (both metrics below state } \\
\text { of Wisconsin benchmarks } \\
\text { of } 7.5 \% \text { and } 13.2 \% \text {, } \\
\text { respectively). } \\
\text { Additionally, time } \\
\text { between hospitalizations } \\
\text { increased. }\end{array}$ & $\begin{array}{l}\text { Additional study measures: Appropriate } \\
\text { medication management (ACE inhibitors \& beta } \\
\text { blockers), LOS, Incorporated meta-analyses, } \\
\text { consensus recommendations, and HF guidelines. } \\
\text { Telephone f/u calls @ } 72 \text { hr, } 1 \text { wk, \& } 2 \text { wk after } \\
\text { DC. } \\
\text { (Intervention: during inpatient stay and } \\
\text { post-discharge) }\end{array}$ \\
\hline $\begin{array}{l}\text { Misky et } \\
\text { al. } \\
(2010)^{[21]}\end{array}$ & $\begin{array}{l}\text { Does timely PCP } \\
\text { f/u affect 30-day } \\
\text { RR? }\end{array}$ & $\begin{array}{l}\text { Prospecti } \\
\text { ve } \\
\text { Cohort } \\
\text { Level IV }\end{array}$ & $\begin{array}{l}\text { Timely PCP } \\
\text { f/u (within } 4 \\
\text { wk after DC). }\end{array}$ & 30-day RR & $\begin{array}{l}\mathrm{N}=65 \text { (PTs } \\
\text { admitted to } \\
\text { general } \\
\text { internal } \\
\text { medicine unit) }\end{array}$ & $\begin{array}{l}\text { Rate of timely PCP f/u } \\
\text { was } 49.2 \% \text {. RR (same } \\
\text { condition) was higher for } \\
\text { PTs lacking timely PCP } \\
\text { f/u ( } 21.2 \% \text { vs. } 3.1 \% ; p \\
=.05 \text { ); RR (readmission } \\
\text { or other care sought for } \\
\text { same condition) was } \\
\text { higher for PTs lacking } \\
\text { timely PCP f/u ( } 28.1 \% \text { vs. } \\
6.3 \% ; p=.02) ; \text { RR (any } \\
\text { condition) did not show } \\
\text { any difference. }\end{array}$ & $\begin{array}{l}\text { Subjects limited to a convenience sample (i.e., } \\
\text { PTs without telephones were excluded) \& may } \\
\text { not be representative of all medical inputs. PCP } \\
\text { f/u and readmission information collected from } \\
\text { PT recollection. } \\
\text { (Intervention: post-discharge) }\end{array}$ \\
\hline $\begin{array}{l}\text { Naylor et } \\
\text { al. } \\
(1999)^{[30]}\end{array}$ & $\begin{array}{l}\text { Will } \\
\text { comprehensive } \\
\text { DC planning } \\
\text { with home visit } \\
\text { f/u by APN for } \\
\text { "at-risk" elders } \\
\text { reduce time to } \\
\text { first } \\
\text { readmission? }\end{array}$ & $\begin{array}{l}\text { RCT } \\
\text { Level II }\end{array}$ & $\begin{array}{l}\text { APN DC } \\
\text { planning, } 2 \\
\text { home visits } \\
\text { (48 hr \& 7-10 } \\
\text { days after } \\
\text { DC), } \\
\text { telephone } \\
\text { availability, \& } \\
\text { weekly } \\
\text { APN-initiated } \\
\text { phone contact. }\end{array}$ & $\begin{array}{l}\text { Time to } \\
\text { first } \\
\text { readmission } \\
\text { for any } \\
\text { reason. }\end{array}$ & $\begin{array}{l}\mathrm{N}=363 \text { (PTs } \\
\text { age } 65 \text { or older } \\
\text { admitted with } \\
1 \text { of top } 10 \\
\text { reasons among } \\
\text { Medicare } \\
\text { population; } \\
\text { meet at least } 1 \\
\text { criteria assoc. } \\
\text { w/poor DC } \\
\text { outcomes } \\
\text { from earlier } \\
\text { study) }\end{array}$ & $\begin{array}{l}\text { Time to first readmission } \\
\text { for any reason was } \\
\text { increased in IG ( } p \\
<.001) \text {. By } 24 \text { wk, CG } \\
\text { more likely to be } \\
\text { readmitted at least once } \\
(37 \% \text { vs. } 20.3 \% ; p \\
<.001) \text {; fewer IG had } \\
\text { multiple readmissions } \\
(6.2 \% \text { vs. } 14.5 \% ; p \\
=.01) ; \text { IG group had } \\
\text { fewer days per PT ( } 1.53 \\
\text { vs. } 4.09, p<.001) .\end{array}$ & $\begin{array}{l}\text { Additional study measures: LOS, \# unscheduled } \\
\text { acute care visits (MD, clinic, ED), cost of } \\
\text { post-index hospital health services, functional } \\
\text { status, depression, patient satisfaction. } \\
\text { (Intervention: during inpatient stay and } \\
\text { post-discharge) }\end{array}$ \\
\hline $\begin{array}{l}\text { Naylor } \\
\& \\
\text { McCaule } \\
\text { y } \\
(1999)^{[32]}\end{array}$ & $\begin{array}{l}\text { Will } \\
\text { comprehensive } \\
\text { DC planning } \\
\text { with home visit } \\
\text { f/u by APN in } \\
\text { PTs with } \\
\text { common medical } \\
\text { \& surgical } \\
\text { cardiac } \\
\text { conditions } \\
\text { decrease } \\
\text { readmissions? } \\
\text { Would the effect } \\
\text { differ between } \\
\text { medical and } \\
\text { surgical cardiac } \\
\text { patients? }\end{array}$ & $\begin{array}{l}\text { RCT } \\
\text { Level II }\end{array}$ & $\begin{array}{l}\text { APN DC } \\
\text { planning } \\
\text { followed by at } \\
\text { least } 2 \text { home } \\
\text { visits (48hr \& } \\
7-10 \text { days after } \\
\text { DC), daily } \\
\text { telephone } \\
\text { availability } \\
\text { (including } \\
\text { weekly } \\
\text { APN-initiated } \\
\text { phone call). }\end{array}$ & $\begin{array}{l}\text { Hospital } \\
\text { readmission } \\
\text { s, } \\
\text { cumulative } \\
\text { days of } \\
\text { rehospitaliz } \\
\text { ation }\end{array}$ & $\begin{array}{l}\mathrm{N}=202 \text { (age } \\
65 \text { or older } \\
\text { hospitalized } \\
\text { with common } \\
\text { medical or } \\
\text { surgical } \\
\text { cardiac } \\
\text { conditions) }\end{array}$ & $\begin{array}{l}\text { Overall: Medical PTs had } \\
\text { more readmissions during } \\
\text { the 24-wk period than } \\
\text { surgical PTs ( } p=.03 \text { ). } \\
\text { Medical sample: } \\
\% \text { medical PTs admitted } \\
\text { at least once in the } 24 \text {-wk } \\
\text { period was similar for IG } \\
\& \text { CG groups; however, } \\
\text { fewer IG had multiple } \\
\text { readmissions ( } p=.05) \\
\text { and total \# of days of } \\
\text { rehospitalization per PT } \\
\text { was less in IG ( } p=.05) \text {. } \\
\text { Surgical sample: } \\
\% \text { surgical PTs admitted } \\
\text { at least once in the } 24 \text {-wk } \\
\text { period was smaller for IG } \\
\text { ( } p=.05) \text {; however, \% of } \\
\text { multiple readmissions } \\
\text { was similar in both } \\
\text { groups. IG had fewer } \\
\text { readmissions from DC to } \\
6 \text { wk }(p=.02), \text { but not } \\
\text { significant from } 6 \text { to } 24 \\
\text { wk ( } p=.06 \text { ). }\end{array}$ & $\begin{array}{l}\text { Additional study measure: functional status. } \\
\text { Study sample drawn from PT cohort in Naylor et } \\
\text { al. large-scale } 1999 \text { RCT. Medical and surgical } \\
\text { subgroups analyzed separately. Of the } 76 \\
\text { medical readmissions, } 51 \text { were for HF PTs. Of } \\
\text { the } 37 \text { surgical readmissions, } 25 \text { were for PTs } \\
\text { who had a CABG during the index hosp. } \\
\text { Overall, most of the readmissions (60\%) were } \\
\text { index related, 21\% related to comorbid } \\
\text { condition, \& } 19 \% \text { for a new health problem. For } \\
\text { medical cardiac PTs, the intervention was most } \\
\text { effective in preventing multiple readmissions, } \\
\text { decreasing the number of hospital days/PT, and } \\
\text { reducing number of hospitalizations with } \\
\text { prolonged LOS. For surgical cardiac PTs, } \\
\text { intervention most effective in preventing early } \\
\text { readmission, decreasing total \# of PTs } \\
\text { readmitted, \& reducing the \# of } \\
\text { rehospitalizations with prolonged LOS. } \\
\text { (Intervention: during inpatient stay and } \\
\text { post-discharge) }\end{array}$ \\
\hline
\end{tabular}

(Table 2 continued on page 43) 
Table 2. (Continued.)

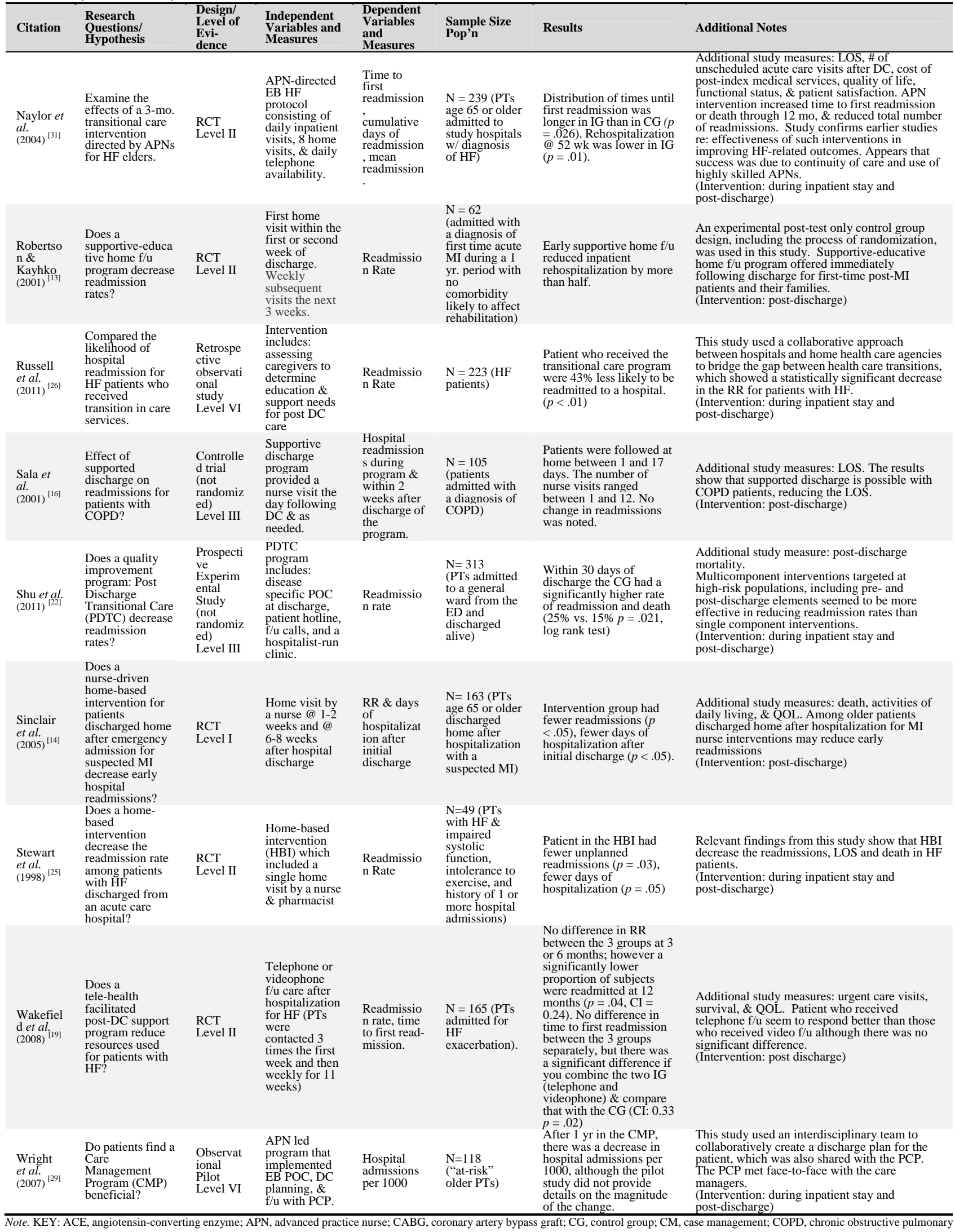
disease; DC, discharge; DM, diabetes mellitus; EB, evidence based; ED, emergency department; f/u, follow-up; GNP, geriatric nurse practitioner; HF, heart failure; HTN, hypertension; IG, intervention group; IH, index hospitalization; LOS, length of stay; MI, myocardial infarction; PCP, primary care provider; POC, plan of care; PT, patient; QOL, quality of life; RCT, randomized controlled trial; RN, registered nurse; RR, readmission rate; \$, health care costs. 


\section{Synthesis of evidence}

The articles were organized in a table format in descending order based on the level of evidence (see Table 3). The data compiled and summarized in the table included detailed information regarding the intervention, the timing of the intervention, readmission rate outcome detail, and whether the intervention was statistically significant in reducing readmissions. By use of this methodology, the data were synthesized and organized into two categories: primary studies in which the readmission was measured as an outcome and studies that systematically reviewed interventions aimed at improving the patient discharge process, with readmissions included as one of the outcome measures.

Table 3. Synthesis of Evidence

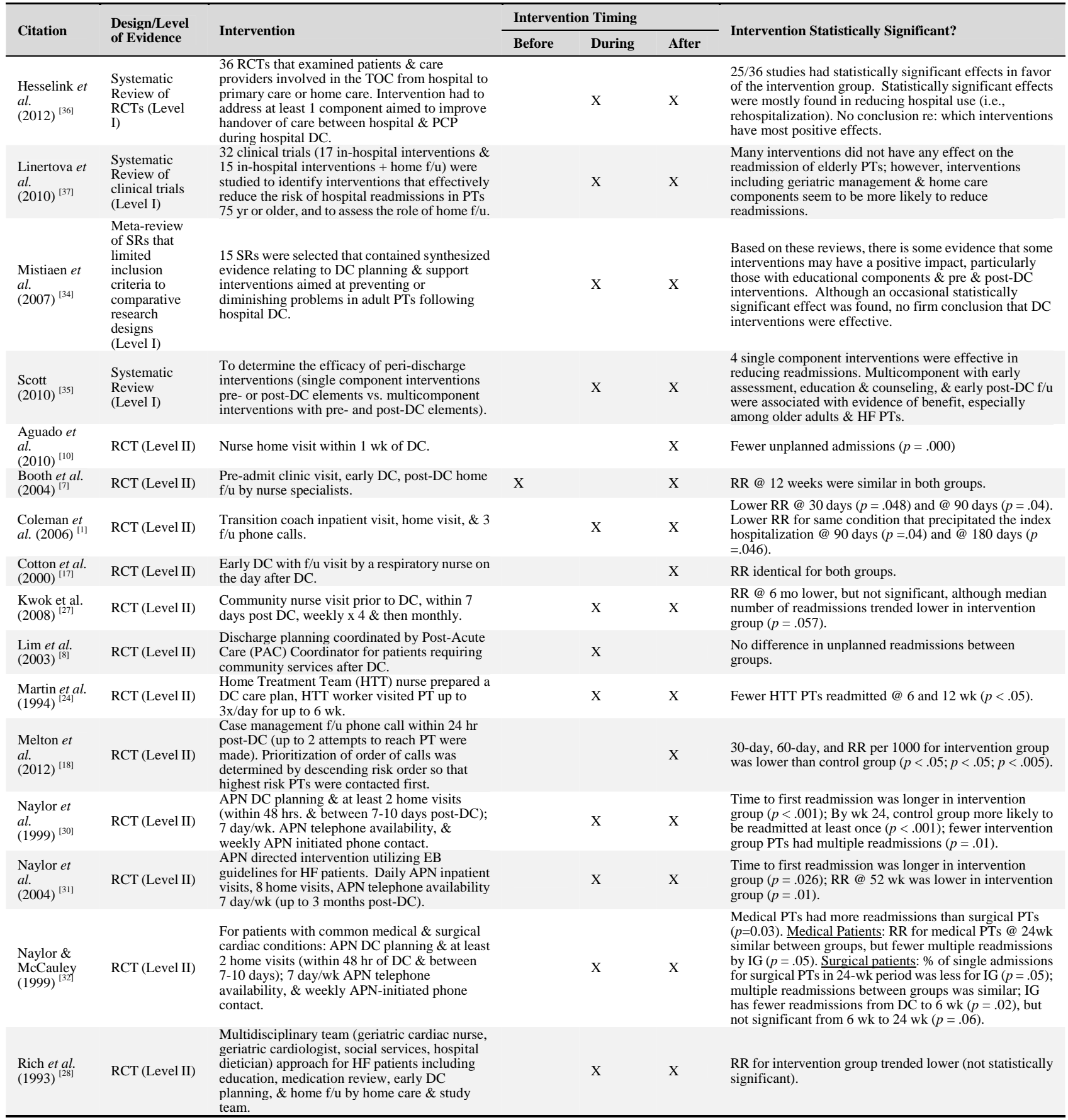

(Table 3 continued on page 45) 
Table 3. (Continued.)

\begin{tabular}{|c|c|c|c|c|c|c|}
\hline \multirow{2}{*}{ Citation } & \multirow{2}{*}{$\begin{array}{l}\text { Design/Level } \\
\text { of Evidence }\end{array}$} & \multirow{2}{*}{ Intervention } & \multicolumn{3}{|c|}{ Intervention Timing } & \multirow{2}{*}{ Intervention Statistically Significant? } \\
\hline & & & Before & During & After & \\
\hline $\begin{array}{l}\text { Robertson } \\
\text { \& Kayhko } \\
(2011)^{[13]}\end{array}$ & RCT (Level II) & $\begin{array}{l}\text { Supportive-educative home f/u for PTs } \\
\text { diagnosis with MI (first time) by a nurse @ } 1^{\text {st }} \\
\text { or } 2^{\text {nd }} \text { wk post-DC followed by weekly } \\
\text { subsequent visits over the next } 3 \text { wk. }\end{array}$ & & & $\mathrm{X}$ & RR for intervention group reduced by half (3 vs. 7 PTs). \\
\hline $\begin{array}{l}\text { Sinclair et } \\
\text { al. } \\
(2005)^{[14]}\end{array}$ & RCT (Level II) & $\begin{array}{l}\text { Home visit f/u by a nurse for PTs with MI @ } \\
\text { 1-2 wk \& 6-8 wk post-DC. }\end{array}$ & & & $\mathrm{X}$ & Fewer readmissions for intervention group $(p<.05)$. \\
\hline $\begin{array}{l}\text { Stewart et } \\
\text { al. } \\
(1998)^{[25]}\end{array}$ & RCT (Level II) & $\begin{array}{l}\text { Home-Based Intervention (HBI) that included } \\
\text { nurse visit before DC, followed by home f/u } \\
\text { visit by nurse \& pharmacist @ } 1 \text { wk post-DC; } \\
\text { also included f/u communication to PCP. }\end{array}$ & & $\mathrm{X}$ & $\mathrm{X}$ & HBI group had fewer readmissions ( $p=.03$ ). \\
\hline $\begin{array}{l}\text { Wakefield } \\
\text { et al. } \\
\text { (2008) }\end{array}$ & RCT (Level II) & $\begin{array}{l}\text { Intervention group was subdivided into } \\
\text { telephone or videophone f/u after } \\
\text { hospitalization for HF. Both groups received } \\
\text { contact } 3 \text { times during the first wk \& then } \\
\text { weekly for } 11 \text { wk. }\end{array}$ & & & $\mathrm{X}$ & $\begin{array}{l}\text { No significant difference in RR @ } 3 \text { \& } 6 \text { mo; however, } \\
\text { significant difference of combined intervention groups } \\
\text { @ } 12 \text { mo }(p=.04) \text {. No difference in time to first } \\
\text { readmission between groups; however, a significant } \\
\text { difference when the two telehealth groups (telephone \& } \\
\text { televideo) were combined }(p=.02) \text {. }\end{array}$ \\
\hline $\begin{array}{l}\text { Coleman et } \\
\text { al. } \\
(2004)^{[33]}\end{array}$ & $\begin{array}{l}\text { Quasi-experim } \\
\text { ental (Level } \\
\text { III) }\end{array}$ & $\begin{array}{l}\text { Through interaction with a Transition Coach } \\
\text { (inpatient visit, home visit, \& } 3 \text { f/u phone calls), } \\
\text { intervention patients received tools to promote a } \\
\text { more active role in their care. }\end{array}$ & & $\mathrm{X}$ & $\mathrm{X}$ & $\begin{array}{l}\text { The odds ratio of readmission in the intervention group = } \\
0.52 \text { [95\% confidence interval }(\mathrm{CI})=0.28-0.96] \text { @ } 30 \\
\text { days; } 0.43(95 \% \mathrm{CI}=0.25-0.72) @ 90 \text { days. }\end{array}$ \\
\hline $\begin{array}{l}\text { Sala et al. } \\
(2001)^{[16]}\end{array}$ & $\begin{array}{l}\text { Controlled } \\
\text { trial, not } \\
\text { randomized } \\
\text { (Level III) }\end{array}$ & $\begin{array}{l}\text { Supported Discharge Program for COPD PTs } \\
\text { which included: use of nebulizers \& continuous } \\
\mathrm{O}_{2} \text { at home, visit by respiratory-trained nurse } \\
\text { day after DC with f/u visits according to PT } \\
\text { needs. PT had access during normal working } \\
\text { hours to nurse. Lung specialist visited PT } \\
\text { before DC from program. }\end{array}$ & & & $\mathrm{X}$ & $\begin{array}{l}\text { Readmission rates while PT in supported discharge } \\
\text { program, within } 2 \text { wk after DC from program, and } \\
\text { greater than } 2 \text { wk after DC from program not statistically } \\
\text { significant between groups. }\end{array}$ \\
\hline $\begin{array}{l}\text { Shu et al. } \\
\text { (2011) }\end{array}$ & $\begin{array}{l}\text { Prospective } \\
\text { Experimental } \\
\text { (non-randomiz } \\
\text { ed) (Level III) }\end{array}$ & $\begin{array}{l}\text { Post-DC transitional care program which } \\
\text { includes: disease-specific care plan @ DC, PT } \\
\text { hotline, scheduled f/u calls, \& a hospitalist-run } \\
\text { clinic. }\end{array}$ & & $\mathrm{X}$ & $\mathrm{X}$ & $\begin{array}{l}\text { Within } 30 \text { days, control group had a significantly higher } \\
\text { rate of readmission \& death }(p=.021 \text {, log rank test). }\end{array}$ \\
\hline $\begin{array}{l}\text { Feldman et } \\
\text { al. } \\
(2011) \\
{[20]}\end{array}$ & $\begin{array}{l}\text { Cohort } \\
\text { Observational } \\
\text { (Level IV) }\end{array}$ & $\begin{array}{l}\text { Intervention included f/u at a multidisciplinary } \\
\text { HF clinic after hospital DC for newly diagnosed } \\
\text { patients. }\end{array}$ & & & $\mathrm{X}$ & $\begin{array}{l}\text { After } 12 \text {-mo of f/u in the HF clinic, } 14.32 \% \text { of patients } \\
\text { were hospitalized (vs. } 29.4 \% \text { within } 6 \text { mo preceding } \\
\text { admission to the clinic). }\end{array}$ \\
\hline $\begin{array}{l}\text { Misky et al. } \\
(2010)^{[21]}\end{array}$ & $\begin{array}{l}\text { Prospective } \\
\text { Cohort } \\
\text { (Level IV) }\end{array}$ & Timely PCP f/u (within 4 wk. post-DC) & & & $\mathrm{X}$ & $\begin{array}{l}49.2 \% \text { of patients had timely PCP } \mathrm{f} / \mathrm{u} \text {. RR for same } \\
\text { condition was higher for PTs lacking timely PCP } \mathrm{f} / \mathrm{u}(p \\
=.05) \text {; RR or other care for same condition was higher } \\
\text { for PTs lacking timely PCP f/u }(p=.02) \text {; RR for any } \\
\text { condition was not statistically significant. }\end{array}$ \\
\hline $\begin{array}{l}\text { Jacob et al. } \\
(2008)^{[38]}\end{array}$ & $\begin{array}{l}\text { Qualitative } \\
\text { Systematic } \\
\text { Review } \\
\text { (Level V) }\end{array}$ & $\begin{array}{l}10 \text { articles (RCTs, quasi-experimental, } \\
\text { retrospective reviews, \& interpretive studies) } \\
\text { that assessed the effects of "enhanced discharge } \\
\text { support" for patients identified as susceptible to } \\
\text { difficult transition when transitioning from } \\
\text { acute hospital back to the community. }\end{array}$ & & $\mathrm{X}$ & $\mathrm{X}$ & $\begin{array}{l}\text { Evidence did indicate support for role of "enhanced } \\
\text { discharge support" in preventing or delaying } \\
\text { readmissions for certain diagnoses, such as HF \& stroke. } \\
\text { In addition, those with adequate social support \& } \\
\text { confidence in self-care tend to experience fewer } \\
\text { readmissions. }\end{array}$ \\
\hline $\begin{array}{l}\text { Bull } \\
(1994)^{[11]}\end{array}$ & $\begin{array}{l}\text { Longitudinal, } \\
\text { Observational } \\
\text { Study } \\
\text { (Level VI) }\end{array}$ & $\begin{array}{l}\text { Elder patients with caregivers discharged home. } \\
\text { Purpose was to describe community services } \\
\text { (skilled \& unskilled home health) used by } \\
\text { elders during } 2 \text { wk. post-DC. }\end{array}$ & & & $\mathrm{X}$ & $\begin{array}{l}\text { Findings suggested that elders who received visiting } \\
\text { nurse (skilled) services are less likely to be readmitted. }\end{array}$ \\
\hline $\begin{array}{l}\text { Cardozo \& } \\
\text { Steinberg } \\
(2010)^{[12]}\end{array}$ & $\begin{array}{l}\text { Observational } \\
\text { Study } \\
\text { (Level VI) }\end{array}$ & $\begin{array}{l}\text { Nurse visit up to } 3 x / \text { wk.; home tele-monitoring } \\
\text { daily. All patients had home-based case } \\
\text { management. }\end{array}$ & & & $\mathrm{X}$ & $\begin{array}{l}60 \text { day RR was } 13.9 \% \text {, which was different than } \\
\text { Outcome Concept System (OCS) national study rate of } \\
56.4 \% \text {. }\end{array}$ \\
\hline $\begin{array}{l}\text { Chang et al. } \\
(2003)^{[9]}\end{array}$ & $\begin{array}{l}\text { Descriptive, } \\
\text { Retrospective } \\
\text { (Level VI) }\end{array}$ & $\begin{array}{l}\text { DC planning program in which RN refers PTs } \\
\text { to DC Planning nurse who interview PT while } \\
\text { in the hospital, and prepares DC plan. }\end{array}$ & & $\mathrm{X}$ & & $\begin{array}{l}\text { 14-day RR related to total \# health professionals visiting } \\
\text { PT not significant }(p>.05) \text {; } 14 \text {-day RR lower when } \\
\text { visited by SW, home care, hospice }(p<.05) \text {; } 14 \text {-day RR } \\
\text { when visited by nutritionists, DM specialists, PT not } \\
\text { significant }(p>.05) \text {. }\end{array}$ \\
\hline $\begin{array}{l}\text { Coleman et } \\
\text { al. } \\
(2005)^{[15]}\end{array}$ & $\begin{array}{l}\text { Descriptive } \\
\text { (Level VI) }\end{array}$ & $\begin{array}{l}\text { GNP visited PTs } 24-48 \mathrm{hr} \text { post-DC to assess the } \\
\text { pre \& post hospitalization medication regimen, } \\
\text { and study the incidence of } \& \text { factors related to } \\
\text { med discrepancies. }\end{array}$ & & & $\mathrm{X}$ & $\begin{array}{l}\text { 30-day RR was higher for PTs experiencing medication } \\
\text { discrepancies }(p=.04) \text {. }\end{array}$ \\
\hline $\begin{array}{l}\text { Miranda et } \\
\text { al. } \\
(2002)^{[23]}\end{array}$ & $\begin{array}{l}\text { Descriptive } \\
\text { (Level VI) }\end{array}$ & $\begin{array}{l}\text { Implementation of EB guidelines for HF } \\
\text { including PT education, \& post-DC telephonic } \\
\text { service for patients across a health system in } \\
\text { Wisconsin. }\end{array}$ & & $\mathrm{X}$ & $\mathrm{X}$ & $\begin{array}{l}\text { Post-implementation results saw a 30-day readmission } \\
\text { rate decrease from } 14 \% \text { to } 6.8 \% \text {, and } 90 \text {-day readmission } \\
\text { rate decrease from } 17.5 \% \text { to } 12.5 \% \text { (both metrics below } \\
\text { state of Wisconsin benchmarks of } 7.5 \% \text { and } 13.2 \% \\
\text { respectively). In addition, results also demonstrated an } \\
\text { increase in time between hospitalizations. }\end{array}$ \\
\hline $\begin{array}{l}\text { Russell et } \\
\text { al. } \\
(2011)^{[26]}\end{array}$ & $\begin{array}{l}\text { Retrospective } \\
\text { Observational } \\
\text { (Level VI) }\end{array}$ & $\begin{array}{l}\text { HF PTs receive transition in care services } \\
\text { (assessing caregivers to determine need for } \\
\text { education \& support, integrating caregivers } \\
\text { with care planning team, \& improving } \\
\text { communication between patient/caregiver \& } \\
\text { PCP. }\end{array}$ & & $\mathrm{X}$ & $\mathrm{X}$ & $\begin{array}{l}\text { Pts. receiving transition in care program were } 43 \% \text { less } \\
\text { likely to be readmitted }(p<.01) \text {. }\end{array}$ \\
\hline $\begin{array}{l}\text { Wright et } \\
\text { al. } \\
(2007)^{[29]}\end{array}$ & $\begin{array}{l}\text { Observational } \\
\text { Pilot (Level } \\
\text { VI) }\end{array}$ & $\begin{array}{l}118 \text { PTs from a RCT were evaluated for } \\
\text { progress of study. Intervention involved } \\
\text { implementation of care management program } \\
\text { for "at-risk" older adults. APN assessed patient } \\
\& \text { assisted with DC planning. Hospital-based } \\
\text { interdisciplinary team generated an EB POC. } \\
\text { After DC, RN Care Manager implemented plan } \\
\text { in collaboration with PCP \& provided f/u. Calls } \\
\text { or visits as needed, even accompanying patient } \\
\text { to f/u PCP appt. }\end{array}$ & & $\mathrm{X}$ & $\mathrm{X}$ & $\begin{array}{l}\text { After } 1 \text { year, showed a decrease in hospital admissions } \\
\text { per } 1000 \text { (pilot did not provide quantification to } \\
\text { determine magnitude of change). }\end{array}$ \\
\hline
\end{tabular}

heart failure; IG, intervention group; LOS, length of stay; MI, myocardial infarction; PCP, primary care provider; POC, plan of care; PT, patient; QOL, quality of life; RCT, randomized controlled trial; RR, readmission rate; SR, systematic review; SW, social worker; TOC, transition of care. 


\subsection{Primary studies addressing readmission as an outcome}

A total of 33 primary study articles examined some measurement of hospital readmission as an outcome. There was considerable heterogeneity among the studies in terms of the types of interventions (timing, setting, population) as well as the type of readmission outcome measured. To answer the clinical question of interest, a decision was made to synthesize and categorize the studies on the basis of the timing of the intervention. The goal was to compare the various intervention timings to determine whether any one category of intervention timing was more effective than another. Following is a summary of these studies categorized by intervention timing.

\subsubsection{I ntervention before and after hospitalization}

Only one study ${ }^{[7]}$ implemented an intervention in which the timing occurred both before and after the patient's hospitalization. Patients were seen in a pre-admit clinic before their hospitalization with a planned goal of early discharge and post-discharge follow-up at home by a nurse specialist. The readmission rate at 12 weeks, although not quantified by the researchers in the study, was stated to be similar in both the control and the intervention groups; thus, the study found no significant difference in the readmission rate for this intervention.

\subsubsection{I ntervention during hospitalization}

Two studies evaluated interventions occurring during the patient's hospital stay only. The first, an RCT ${ }^{[8]}$, implemented a program in which a Post-Acute Care coordinator provided discharge planning for patients requiring community services after discharge. No differences in unplanned readmissions were noted. The second study ${ }^{[9]}$, a retrospective, descriptive study, analyzed 14-day readmission and risk factor data for patients who were visited by a discharge planning coordinator during hospitalization. The 14-day unplanned readmission rate was significantly greater for patients visited by social workers, home-care nurses, and hospice home-care nurses $(p<.05)$ versus other types of health care providers (e.g., physical therapists, nutritionists, and diabetes education specialists). The researchers concluded that this might be a reflection of the severity of illness of the patients requiring home health services from home-care and hospice nurses.

\subsubsection{I ntervention after hospitalization}

Home follow-up is an alternative way of promoting health with an aim at decreasing readmission rates. The following 12 studies evaluated the effectiveness of an intervention that was implemented after the patient was discharged from the acute care facility. The interventions evaluated in these studies included: a nurse home visit, early discharge of the patient followed by a nurse home visit, case management follow-up phone call, post-discharge telephone call by a nurse, videophone follow-up, and follow-up support to help patients make appointments in primary care clinics after discharge. Of these 12 studies, 7 demonstrated statistically significant results at decreasing the readmission rate and 5 did not.

Many of the studies involved a nurse home visit after discharge. Five of the studies ${ }^{[10-14]}$ saw either a trend towards decreased readmission rates or a statistically significant drop $\left(p=.0^{[10]}, p<.05^{[14]}\right)$ in readmissions when a nurse visited patients in their homes after discharge. All five of these studies provided a nurse home visit within the first 2 weeks after discharge, with several of them providing more than one visit.

Another study ${ }^{[15]}$ involved a geriatric nurse home visit within 72 hours of discharge. A comprehensive medication assessment of medication usage and adherence before and after hospitalization was performed during that visit. The number of discrepancies was categorized by using a medication discrepancy tool. Patients experiencing medication discrepancies had a higher rate of rehospitalization than did those with no discrepancies $(p=.04)$.

Chronic obstructive pulmonary disease (COPD) patients receiving home follow-up visits by a respiratory-trained nurse were evaluated in two other studies. The intervention in the first study ${ }^{[16]}$ included a post-discharge home visit by a respiratory-trained nurse, whereas the other study's ${ }^{[17]}$ intervention evaluated early discharge and home follow-up by a nurse. Although these two studies demonstrated a decreased number of total hospital days used over 12 months ${ }^{[16]}$ and 60 days ${ }^{[17]}$, neither saw a change in the rate of readmission when patients were discharged home and provided a home visit by a specially trained respiratory nurse. 
Post-discharge telephone follow-up within 24 hours by a case manager was the focus of another study ${ }^{[18]}$. Prioritization was made on the order of calls, so that patients at highest risk of readmission were called first. The 30-day and 60-day readmission rate per 1000 was significantly lower with this intervention than in the control group $(p=.01)$. The case manager provided the patient with some direction on how and where to follow up after they were discharged from the acute care facility.

Interventions that provided post-discharge follow-up through the use of a telephone or videophone were evaluated in two studies and demonstrated effectiveness in reducing readmission rates. One study ${ }^{[19]}$ divided patients into two intervention groups (telephone follow-up and video follow-up) and compared these groups with the control group both separately and together. Both intervention groups received contact three times during the first week and then weekly for 11 weeks. There was no significant difference in the readmission rate of these patients at 3 and 6 months; however, there was a significant difference at 12 months when combining the two intervention groups $(p=.04)$. The second study ${ }^{[12]}$ involved daily tele-monitoring in addition to a nurse home visit up to three times weekly and compared outcomes with those obtained in a previous large national tele-health study. This study resulted in a lower 60-day readmission rate than that found in a previous, large national study (13.9\% versus $56.4 \%)$.

The impact of PCP follow-up after discharge on readmission rates was evaluated in two studies. Patients in the first study ${ }^{[20]}$ were followed in a multidisciplinary heart failure clinic for 12 months after discharge from an acute care facility with a new diagnosis of heart failure. By using linear regression, the study found that patients who were followed closely through a heart failure clinic had a significant decrease in use of hospital services as measured by emergency department visits and hospital admissions $(p<.05)$. The second study ${ }^{[21]}$ evaluated the effect on readmission rates when patients had timely (within 4 weeks) follow-up with their PCP after discharge. Only $49.2 \%$ of the patients studied had timely follow-up. Higher readmission rates for the same medical condition were seen in the patients without timely PCP follow-up $(p=.05)$. However, hospital readmission for any condition did not differ with the absence of timely PCP follow-up.

\subsubsection{I ntervention during and after hospitalization}

Thirteen articles had multicomponent interventions that were timed during hospitalization and after discharge from an acute care facility. Although they all had "during" and "after" hospital components, the difference among them was in the type and number of elements that bridged the transition from one level of care to another. Nine of the articles reported statistically significant decreases in the readmission rate and four reported not significant but a trend toward decreases in the readmission rate.

Two studies provided an intervention consisting of an inpatient visit followed by a phone call follow-up after discharge. One such study ${ }^{[22]}$ provided the intervention group with a Post Discharge Transitional Care (PDTC) program that included a disease-specific care plan at discharge, patient access to a hotline, scheduled follow-up phone calls, and access to a clinic run by a hospitalist if needed. At 30 days the control group had a significantly higher readmission rate than did the intervention group $(p=.021)$. Miranda et al. ${ }^{[23]}$ evaluated the implementation of a cross-site evidence-based approach to heart failure within a large health system in southeast Wisconsin. Disease-specific management guidelines, patient education, and post-discharge telephone follow-up calls were implemented system-wide for heart failure patients. Post-implementation results saw the 30 -day readmission rate decrease from $14 \%$ to $6.8 \%$ and the 90 -day readmission rate decrease from $17.5 \%$ to $12.5 \%$ (both metrics were below the state of Wisconsin benchmarks of $7.5 \%$ and $13.2 \%$, respectively). In addition, on the basis of the readmission ratio (number of repeat encounters in both 30 and 90 days), the results also demonstrated an increase in time between hospitalizations when patients were provided this intervention.

An inpatient visit followed by some type of home visit was the intervention evaluated in five studies. Three of the studies proved to be statistically significant in decreasing readmission rates. The first study ${ }^{[24]}$ used a Home Treatment Team (HTT) to prepare a discharge plan for the patient followed by HTT worker visits to the patient up to three times a day for as long as 6 weeks. Fewer HTT patients than control patients were readmitted at 6 and 12 weeks $(p<.05)$. The intervention in the second study ${ }^{[25]}$ was a Home Based Intervention (HBI) that included a nurse visit before the patient was discharged, 
and a nurse and pharmacist home visit within 1 week after discharge. This intervention also included follow-up communication with the patient's PCP. During the 6-month follow-up period, patients in the HBI group had fewer unplanned readmissions $\left(p=.03\right.$ ). A third study ${ }^{[26]}$, a retrospective, observational study, evaluated the likelihood of hospital readmission for heart failure patients who had received transitional care services. Using a logistic regression equation, the odds ratio of 30-day readmission among intervention patients was $0.57(p<.01)$. The other two studies ${ }^{[27,28]}$ saw a decrease in the readmission rate for the intervention group at 12 months ${ }^{[27]}$ and 90 days ${ }^{[28]}$; however, the decrease was not statistically significant.

An observational pilot study ${ }^{[29]}$, which was done from a larger RCT, implemented a care management program for 118 at-risk older adults. An advanced practice nurse assessed the patient and assisted with the discharge plan. A hospital-based interdisciplinary team generated a care plan by using evidence-based protocols. After discharge, the registered nurse care manager implemented the plan in collaboration with the PCP and provided follow-up consisting of phone calls or home visits as needed. Twelve months after implementation of the program, there was a decrease in hospital admissions per 1000, although the pilot study did not provide details on the magnitude of the change.

Several studies led by researchers well known for work in transitional care were included in this review. Although slightly different, both of these researchers frame their interventions similarly: patient visits and discharge planning during hospitalization, home visits, and phone follow-up or availability after discharge. Three of these studies, led by Mary Naylor ${ }^{[30-32]}$, evaluated an advanced practice nurse-directed comprehensive discharge planning intervention for three different populations: older patients admitted with a variety of diagnoses commonly seen in Medicare patients, patients diagnosed with heart failure, and older patients hospitalized with common medical and surgical cardiac conditions. Patients received inpatient visits, home visits, weekly phone contact, and phone accessibility to advanced practice nurses. One study ${ }^{[30]}$ demonstrated that patients in the control group were more likely to be readmitted within 24 weeks $(p<.001)$ and that patients receiving the interventions had fewer multiple readmissions $(p=.01)$. A second study ${ }^{[31]}$ evaluated the advanced practice nurse-directed comprehensive discharge planning in heart failure patients and found that readmission rates at 52 weeks were lower in the intervention group $(p=.047)$. Naylor also completed a secondary analysis ${ }^{[32]}$ comparing readmission data between patients with common medical and surgical cardiac conditions. Overall, this analysis found that medical patients experienced more readmissions during the 24-week period than did surgical patients $(p=.03)$, and intervention patients in both groups (medical and surgical) had fewer readmissions. All three studies also demonstrated increased time to first readmission among intervention patients.

Another prominent researcher, Eric Coleman ${ }^{[1,33]}$, implemented a care transitions intervention (CTI) in which patients interacted with a transition coach during hospitalization and after discharge. One study ${ }^{[33]}$ compared patients receiving CTI with administrative data derived from a comparison group. The odds ratio comparing rehospitalization between the two groups demonstrated that intervention patients were approximately half as likely to return to the hospital as was the control group at 30 days $(0.52$; 95\% confidence interval $[\mathrm{CI}]=0.28-0.96)$, 90 days $(0.43 ; 95 \% \mathrm{CI}=0.25-0.72)$, and 180 days $(0.57 ; 95 \% \mathrm{CI}=0.36-0.92)$. Coleman conducted a second study ${ }^{[1]}$ using an RCT design and found similar results: intervention patients had lower rehospitalization rates than did the control group at 30 days $(p=.048)$ and at 90 days $(p$ $=.04)$. Coleman's CTI differed from other interventions in that the role of the transition coach was designed to be supportive with a focus on coaching patients by using self-care skills and tools to better manage their own care for current and future care transitions.

In synthesizing and evaluating the components and timing of the interventions, we found that no intervention resulted in a higher readmission rate. In all of these studies, the readmission rate stayed the same, trended better, or was statistically significant in favor of the intervention group. In summary, among the various intervention timings, 9 of 13 studies evaluating an intervention occurring during and after hospitalization demonstrated significant results. This may indicate that implementing a multicomponent transitional care plan that spans from hospital to home may be of benefit for patients. 


\subsection{Systematic reviews of interventions to improve the discharge process}

Two meta-reviews, two systematic reviews, and one qualitative systematic review met the inclusion criteria and served as an additional source of data for this analysis. All these reviews had a common purpose, which was to examine the effectiveness of discharge interventions or services for patients transitioning from hospital to home. The date range of these reviews was from 2002 to 2012 with a search period spanning from 1977 to 2011.

Two meta-reviews evaluated reviews examining the efficacy of discharge interventions. One meta-review ${ }^{[34]}$ included 15 systematic reviews of comparative research design studies (265 primary studies) and had to deal with considerable heterogeneity, thus making synthesizing and pooling the data difficult. Only 11 of the reviews measured readmission data with 7 reporting inconclusive results and 1 showing a positive but non-significant trend. The three remaining reviews reported a positive effect, especially when the interventions were provided both at the hospital and at home and included an educational component. The second meta-review ${ }^{[35]}$ evaluated seven systematic reviews of controlled trials, and the number of primary studies included in this review was unclear. Discharge interventions in this review were categorized into two groups for analysis: single-component interventions timed before or after discharge and multicomponent interventions timed before and after discharge. Several multicomponent strategies demonstrated positive outcomes in reducing readmissions. Although neither meta-review was able to reach any firm conclusions regarding the effectiveness of discharge interventions, both suggested that multicomponent interventions spanning the transition from hospital to home tend to lead to the greatest effects.

The two systematic reviews shared a similar objective of systematically reviewing interventions that strive to improve patient discharge from hospital to home. Hesselink et al. ${ }^{[36]}$ included 36 studies (all RCTs) of mostly elderly patients with various diagnoses. Multicomponent interventions, used in 34 of 36 studies, were categorized by intervention timing (during hospitalization, at discharge, after discharge, a combination spanning two time periods, a combination spanning all three time periods, or unclear timing). Hospital use outcomes, which included readmission measurements, were evaluated in 20 studies, and half of them had statistically significant results. In their review, Linertova et al. ${ }^{[37]}$ included 32 studies of clinical trials (randomized and nonrandomized) aimed to reduce readmissions in elderly patients. These studies were divided into two groups: in-hospital intervention only $(\mathrm{n}=17)$ and in-hospital intervention with home follow-up $(\mathrm{n}=15)$. A significant effect on the readmission outcome was demonstrated in three studies from the in-hospital intervention group and in seven studies from the interventions with home follow-up group. Both of these systematic reviews concluded that many interventions, especially those that include home care components, seem to have positive effects; however, it was not possible to reach firm conclusions regarding which interventions have these effects.

The 2008 qualitative systematic review by Jacob and Poletick ${ }^{[38]}$ included 10 studies (RCTs, quasi-experimental, retrospective, and interpretative studies) exploring patient experiences with the transition process from hospital to home, including interventions, diagnoses, and patient characteristics. The 10 studies were grouped into one of two categories: interventions or patient characteristics. Interventions included discharge preparation, discharge support, or a combination of both. Patient characteristics included demographics, diagnoses, or other characteristics that may predict successful transitions. Successful transition was defined by reduced readmissions, emergency department use, and mortality. Five studies fell into the intervention category, and three were significant for successful transition, especially for certain diagnoses such as heart failure and stroke. The other two studies trended toward a successful transition but were not significant. In addition, this review also found that patients with adequate social support and confidence in self-care tend to experience fewer readmissions than those living alone and who perceive themselves as not ready to return home.

\section{Summary}

The majority of the studies involved medically complex elderly patients, with some studies limiting their study group to a specific diagnosis (e.g., heart failure, COPD). The articles reviewed provided clear evidence that the current discharge 
process is not adequate for medically complex and older adults to safely re-enter the community after discharge. In all but one of the studies reviewed, a transitional care intervention resulted in a statistically significant reduction in the readmission rate, or a rate trending lower, or the rate remained the same. The only study that found a higher readmission rate in the intervention group included patients who received post-discharge home visits by hospice home-care nurses. The severity of illness of these patients may have contributed to the higher readmission rate.

Multidisciplinary, multicomponent interventions, especially those that include pre- and post-discharge components, seemed to have positive effects. In most of the studies, nursing played a significant role in leading or coordinating the patient's transitional care. Common interventions implemented during hospitalization found in many of the studies included some type of patient assessment process to identify at-risk patients, such as implementation of an evidence-based plan of care; integration of caregivers into the discharge process; multidisciplinary, collaborative planning; and coordination of handoffs to the PCP and community services. Post-discharge interventions included nursing assessments of the patient's home environment, caregiver support, and psychosocial status; accurate medication reconciliation; home or phone follow-up; appropriate community service referrals as needed; timely PCP follow-up; and coaching support to engage and empower patients in effectively self-managing their health.

\section{Limitations}

This analysis had several limitations. First, it was not an all-inclusive review of articles that explore the effectiveness of transitional care interventions. This review was intended to analyze data from studies focusing on single or multicomponent interventions and to determine whether any conclusions could be drawn from these studies that might positively impact readmission rates. Many of the studies also evaluated other outcomes such as mortality, functional outcomes, community services used, and overall patient well-being. Therefore, some of the interventions studied demonstrated effectiveness in terms of these other outcomes apart from the readmission rate. Second, this qualitative systematic review included both quantitative and qualitative studies. Many of the RCTs and other well-designed studies provided evidence in favor of some type of transition of care intervention, and although not as relevant in answering the clinical question, some of the descriptive and qualitative studies provided valuable information to the review. Third, the way the studies were categorized and grouped according to the timing of the intervention for the purpose of analysis may have simplified or ignored important differences between the studies. For example, some studies were grouped because the intervention involved a follow-up phone call; however, this review did not go into detail regarding how many follow-up phone calls were provided to the patients in each study. This was a risk of categorization when evaluating complex interventions, but the group felt that the scheme was fair to each study that was included.

\section{Recommendations for practice}

Transitional care models present many opportunities to both hospitals and community-based organizations to improve the quality of care for complex patients. The evidence demonstrates that there is value in reconfiguring the current discharge processes toward interventions that are more likely to reduce readmissions. The data suggest that a formal transition of care program involving patients and caregivers during the acute hospitalization with follow-up after discharge reduces the risk of readmission. Nursing needs to take the lead in developing and implementing the transition of care plan and in educating patients, caregivers, and the interdisciplinary team.

Health care is rich in evidence-based innovations, yet even when implemented successfully in one location, the same innovation may be disseminated slowly or not at all in other locations. Diffusion of innovations is a major challenge in all industries, including health care ${ }^{[39]}$. Change models outlining how change takes place within an organization are being adopted in health care to maintain scientific and clinical progress. To ensure that change in health care continues to move forward, leaders, researchers, and practitioners are needed who understand and embrace translation of new science, its application, and its evaluation ${ }^{[40]}$. 


\section{References}

[1] Coleman EA, Parry C, Chalmers S, Min SJ. The care transitions intervention: results of a randomized controlled trial. Arch Intern Med. 2006; 166(17): 1822-1828. http://dx.doi.org/10.1001/archinte.166.17.1822

[2] Health Care Advisory Board. High-risk patient care management: Prioritizing high-value opportunities for managing total cost of care [Internet]. Washington (DC): The Advisory Board Company; 2012. Available from:

http://www.advisory.com/Research/Health-Care-Advisory-Board/Studies/2012/High-Risk-Patient-Care-Management

[3] Centers for Medicare and Medicaid Services. MedPAR data [Internet]. Baltimore (MD): CMS; 2013. Available from: http://www.cms.gov/Research-Statistics-Data-and-Systems/Statistics-Trends-and-Reports/MedicareFeeforSvcPartsAB/MEDPA R.html.

[4] Medicare Payment Advisory Commission (Medpac). Report to the Congress: Medicare payment policy [Internet]. Washington (DC): Medpac; 2012. Available from: www.medpac.gov/documents/Mar12_EntireReport.pdf.

[5] Chugh A, Williams MV, Grigsby J, Coleman EA. Better transitions: improving comprehension of discharge instructions. Front Health Serv Manage. 2009 Spring; 25(3): 11- 32. PMid:19382514

[6] Boling PA. Care transitions and home health care. Clin Geriatr Med. 2009; 25: 135-148.

[7] Booth JE, Roberts JA, Flather M, Lamping DL, Mister R, Abdalla M, et al. A trial of early discharge with homecare compared to conventional hospital care for patients undergoing coronary artery bypass grafting. Heart (British Cardiac Society). 2004; 90(11): 1344-1345. http://dx.doi.org/10.1136/hrt.2003.024323

[8] Lim WK, Lambert SF, Gray LC. Effectiveness of case management and post-acute services in older people after hospital discharge. M J Aust. 2003; 178(6): 262-266. Available from: http://www.crd.york.ac.uk/crdweb/ShowRecord.asp?LinkFrom=OAI\&ID=22003000709

[9] Chang SH, Chiu YH, Liou IP. Risks for unplanned hospital readmission in a teaching hospital in southern Taiwan. Int J Nurs Pract. 2003; 9(6): 389-395. http://dx.doi.org/10.1046/j.1440-172X.2003.00443.x

[10] Aguado O, Morcillo C, Delas J, Rennie M, Bechich S, Schembari A, et al. Long-term implications of a single home-based educational intervention in patients with heart failure. Heart Lung. 2010; 39(6 Suppl): S14-22. http://dx.doi.org/10.1016/j.hrtlng.2010.04.010

[11] Bull MJ. Use of formal community services by elders and their family caregivers 2 weeks following hospital discharge. J Adv Nurs. 1994; 19(3): 503-508.

[12] Cardozo L, Steinberg J. Telemedicine for recently discharged older patients. Telemedicine J E Health. 2010; 16(1): 49-55. http://dx.doi.org/10.1089/tmj.2009.0058

[13] Robertson KA, Kayhko K. Cost analysis of an intensive home follow-up program for first-time post-myocardial infarction patients and their families. Off J Can Assoc Crit Care Nurs. 2001; 12(4): 25-31. Available from: http://www.crd.york.ac.uk/crdweb/ShowRecord.asp?LinkFrom=OAI\&ID=22002007552

[14] Sinclair AJ, Conroy SP, Davies M, Bayer AJ. Post-discharge home-based support for older cardiac patients: a randomised controlled trial. Age Ageing. 2005; 34(4): 338-343. http://dx.doi.org/10.1093/ageing/afi116

[15] Coleman EA, Smith JD, Raha D, Min SJ. Posthospital medication discrepancies: prevalence and contributing factors. Arch Int Med. 2005; 165(16): 1842-1847.

[16] Sala E, Alegre L, Carrera M, Ibars M, Orriols FJ, Blanco ML, et al. Supported discharge shortens hospital stay in patients hospitalized because of an exacerbation of COPD. Eur Respir J. 2001; 17(6): 1138-1142. Available from: http://taiss.com/gepoc/gecarp/gepubli/publdoc/109-141-salae-supported-discharge-shortens-hospital-stay.pdf PMid:11491156 http://dx.doi.org/10.1183/09031936.01.00068201

[17] Cotton MM, Bucknall CE, Dagg KD, Johnson MK, MacGregor G, Stewart C, Stevenson RD. Early discharge for patients with exacerbations of chronic obstructive pulmonary disease: a randomized controlled trial. Thorax. 2000; 55(11): 902-906. http://dx.doi.org/10.1136/thorax.55.11.902

[18] Melton LD, Foreman C, Scott E, McGinnis M, Cousins M. Prioritized post-discharge telephonic outreach reduces hospital readmissions for select high-risk patients. Am J Manag Care. 2012; 18(12): 838-844. Available from: http:/www.ajmc.com/publications/issue/2012/2012-12-vol18-n12/Prioritized-Post-Discharge-Telephonic-Outreach-Reduces-Ho spital-Readmissions-for-Select-High-Risk-Patients PMid:23286612

[19] Wakefield BJ, Ward MM, Holman JE, Ray A, Scherubel M, Burns TL, et al. Evaluation of home telehealth following hospitalization for heart failure: a randomized trial. Telemed J E Health. 2008; 14(8):753-761. http://dx.doi.org/10.1089/tmj.2007.0131

[20] Feldman DE, Ducharme A, Giannetti N, Frenette M, Michel C, Grondin F, et al. Outcomes for woman and men who attend a heart failure clinic: results of a 12-month longitudinal study. J Cardiac Fail. 2011; 17(7): 540-546. http://dx.doi.org/10.1016/j.cardfail.2011.03.001 
[21] Misky GJ, Wald HL, Coleman EA. Post-hospitalization transitions: examining the effects of timing of primary care provider follow-up. J Hosp Med. 2010; 5(7): 392-397. http://dx.doi.org/10.1002/jhm.666

[22] Shu CC, Hsu NC, Lin YF, Wang JY, Lin JW, Ko WJ. Integrated postdischarge transitional care in a hospitalist system to improve discharge outcome: an experimental study. BMC Med. 2011; 9: 96. http://dx.doi.org/10.1186/1741-7015-9-96

[23] Miranda MB, Gorski LA, LeFevre JG, Levac KA, Niederstadt JA, Toy AL. An evidence-based approach to improving care of patients with heart failure across the continuum. J Nurs Care Qual. 2002; 17(1): 1-14. Available from: http://journals.lww.com/jncqjournal/Abstract/2002/10000/An_Evidence_Based_Approach_to_Improving_Care_of.2.aspx PMid:12369744 http://dx.doi.org/10.1097/00001786-200210000-00002

[24] Martin F, Oyewole A, Moloney A. A randomized controlled trial of a high support hospital discharge team for elderly people. Age Ageing. 1994; 23(3): 228-234. http://dx.doi.org/10.1093/ageing/23.3.228

[25] Stewart S, Pearson S, Horowitz JD. Effects of a home-based intervention among patients with congestive heart failure discharged from acute hospital care. Arch Int Med. 1998; 158(10): 1067-1072. http://dx.doi.org/10.1001/archinte.158.10.1067

[26] Russell D, Rosati RJ, Sobolewski S, Marren J, Rosenfeld P. Implementing a transitional care program for high-risk heart failure patients: Findings from a community-based partnership between a certified home healthcare agency and regional hospital. J Healthc Qual. 2011; 33(6): 17-23; quiz 23-4. http://dx.doi.org/10.1111/j.1945-1474.2011.00167.x

[27] Kwok T, Lee J, Woo J, Lee DT, Griffith S. A randomized controlled trial of a community nurse-supported hospital discharge programme in older patients with chronic heart failure. J Clin Nurs. 2008; 17(1): 109-117.

[28] Rich MW, Vinson JM, Sperry JC, Shah AS, Spinner LR, Chung MK, Davila-Roman V. Prevention of readmission in elderly patients with congestive heart failure: results of a prospective, randomized pilot study. J Gen Intern Med. 1993; 8(11): 585-590. http://dx.doi.org/10.1007/BF02599709

[29] Wright K, Hazelett S, Jarjoura D, Allen K. The AD-LIFE trial: Working to integrate medical and psychosocial care management models. Home Healthc Nurse. 2007; 25(5): 308-314. http://dx.doi.org/10.1097/01.NHH.0000269964.34045.d5

[30] Naylor MD, Brooten D, Campbell R, Jacobsen BS, Mezey MD, Pauly MV, Schwartz JS. Comprehensive discharge planning and home follow-up of hospitalized elders: a randomized clinical trial. JAMA. 1999; 281(7): 613-620.

http://dx.doi.org/10.1001/jama.281.7.613

[31] Naylor MD, Brooten DA, Campbell RL, Maislin G, McCauley KM, Schwartz JS. Transitional care of older adults hospitalized with heart failure: a randomized, controlled trial. J Am Geriatr Soc. 2004; 52(5): 675-684.

http://dx.doi.org/10.1111/j.1532-5415.2004.52202.x

[32] Naylor MD, McCauley KM. The effects of a discharge planning and home follow-up intervention on elders hospitalized with common medical and surgical cardiac conditions. J Cardiovasc Nurs. 1999; 14(1): 44-54. Available from: http://www.ncbi.nlm.nih.gov/pubmed/10533691 PMid:10533691 http://dx.doi.org/10.1097/00005082-199910000-00006

[33] Coleman EA, Smith JD, Frank JC, Min SJ, Parry C, Kramer AM. Preparing patients and caregivers to participate in care delivered across settings: the care transitions intervention. J Am Geriatr Soc. 2004; 52(11): 1817-1825.

http://dx.doi.org/10.1111/j.1532-5415.2004.52504.x

[34] Mistiaen P, Francke AL, Poot E. Interventions aimed at reducing problems in adult patients discharged from hospital to home: a systematic meta-review. BMC Health Serv Res. 2007; 7: 47. http://dx.doi.org/10.1186/1472-6963-7-47

[35] Scott IA. Preventing the rebound: Improving care transition in hospital discharge processes. Aust Health Rev. 2010; 34(4): 445-451. http://dx.doi.org/10.1071/AH09777

[36] Hesselink G, Schoonhoven L, Barach P, Spijker A, Gademan P, Kalkman C, Liefers J, Vernooij-Dassen M, Wollersheim H. Improving patient handovers from hospital to primary care: A systematic review. Ann Intern Med. 2012; 157: 417-428. Available from: http://www.handover.eu/upload/library/net9gfc2955r4g7declv1.pdf PMid:22986379 http://dx.doi.org/10.7326/0003-4819-157-6-201209180-00006

[37] Linertova R, Garcia-Perez L, Vazquez-Diaz JR, Lorenzo-Riera A, Sarria-Santamera A. Interventions to reduce hospital readmissions in the elderly: In-hospital or home care. A systematic review. J Eval Clin Pract. 2011; 17(6): 1167-1175. http://dx.doi.org/10.1111/j.1365-2753.2010.01493.x

[38] Jacob L, Poletick EB. Systematic review: predictors of successful transition to community-based care for adults with chronic care needs. Care Manag J. 2008; 9(4): 154-165. http://dx.doi.org/10.1891/1521-0987.9.4.154

[39] Berwick DM. Disseminating innovations in health care. J Am Med Assoc. 2003; 289(15): 1969-1975. http://dx.doi.org/10.1001/jama.289.15.1969

[40] White KM, Dudley-Brown S. Translation of evidence into nursing and health care practice. New York (NY): Spring Publishing Co; 2012. 